\title{
Quiescent Giant Molecular Cloud Cores in the Galactic Center
}

D. Lis, E. Serabyn, R. Zylka \& Y. Li

$2000-14$

To Appear in

The Astrophysical Journal 



\title{
Quiescent Giant Molecular Cloud Cores in the Galactic Center
}

\author{
D.C. Lis ${ }^{1}$, E. Serabyn ${ }^{1}$, R. Zylka ${ }^{2}$, and Y. Li ${ }^{1}$
}

\begin{abstract}
We have used the Long Wavelength Spectrometer (LWS) aboard the Infrared Space Observatory (ISO) to map the far-infrared continuum emission (45-175 $\mu \mathrm{m}$ ) toward several massive Giant Molecular Cloud (GMC) cores located near the Galactic center. The observed far-infrared and submillimeter spectral energy distributions imply low temperatures $(\sim 15-22 \mathrm{~K})$ for the bulk of the dust in all the sources, consistent with external heating by the diffuse ISRF and suggest that these GMCs do not harbor highmass star-formation sites, in spite of their large molecular mass. Observations of FIR atomic fine structure lines of $\mathrm{CI}$ and $\mathrm{OI}$ indicate an ISRF enhancement of $\sim 10^{3}$ in the region. Through continuum radiative transfer modeling we show that this radiation field strength is in agreement with the observed FIR and submillimeter spectral energy distributions, assuming primarily external heating of the dust with only limited internal luminosity $\left(\sim 2 \times 10^{5} \mathrm{~L}_{\odot}\right)$. Spectroscopic observations of millimeter-wave transitions of $\mathrm{H}_{2} \mathrm{CO}, \mathrm{CS}$, and $\mathrm{C}^{34} \mathrm{~S}$ carried out with the Caltech Submillimeter Observatory (CSO) and the Institut de Radio Astronomie Millimétrique (IRAM) 30-meter telescope indicate a gas temperature of $\sim 80 \mathrm{~K}$, significantly higher than the dust temperatures, and density of $\sim 1 \times 10^{5} \mathrm{~cm}^{-3}$ in GCM0.25+0.01, the brightest submillimeter source in the region. We suggest that shocks caused by cloud collisions in the turbulent interstellar medium in the Galactic center region are responsible for heating the molecular gas. This conclusion is supported by the presence of wide-spread emission from molecules such as $\mathrm{SiO}$, $\mathrm{SO}$, and $\mathrm{CH}_{3} \mathrm{OH}$, which are considered good shock tracers. We also suggest that the GMCs studied here are representative of the "typical", pre-starforming cloud population in the Galactic center.
\end{abstract}

Subject headings: Galaxy: center - ISM: clouds - ISM: continuum - ISM: individual (GCM0.25+0.11, Sgr B1) - stars: formation

\footnotetext{
${ }^{1}$ California Institute of Technology, Downs Laboratory of Physics 320-47, Pasadena, CA 91125

${ }^{2}$ Institut für Theoretische Astrophysik, Universität Heidelberg, Tiergartenstr. 15, D-69121 Heidelberg, Germany
} 


\section{Introduction}

The harsh environment in the Galactic center region (central $\sim 200 \mathrm{pc}$ of the Milky Way; the region referred to as the central molecular zone; see reviews by Morris \& Serabyn 1996 and Mezger et al. 1996), characterized by disruptive tidal forces, high gas pressures (Bally et al. 1988; Spergel \& Blitz 1992) and strong magnetic fields (Morris 1989) may suppress gravitational collapse in all but the most massive clouds. Consequently, star formation there may be caused primarily by shock compression due to cloud-cloud collisions, resulting in a flatter initial mass function than in the Galactic disk (Morris 1993). Recent NIR imaging and spectroscopic observations have revealed several new high-mass star-formation sites in the Galactic center. In excess of 100 main sequence stars with masses up to $120 \mathrm{M}_{\odot}$ have been detected in the Arches and Quintuplet clusters, the most massive young clusters in the Galaxy (Serabyn et al. 1998; Figer et al. 1999). In particular, the HST observations of Figer et al. (1999) allow detection of main sequence stars with initial masses well below $10 \mathrm{M}_{\odot}$. The resulting IMF in the two clusters has a slope significantly more positive than the average for young clusters elsewhere in the Galaxy ( $\Gamma=-0.65$ compared to -1.4$)$.

Compact, massive clusters thus clearly form in the central region. However, as discussed by Serabyn et al. (1998) and Figer et al. (1999), their characteristics suggest a formation mechanism substantially different from that for the disk clusters, presumably a more violent one. We might expect to see many more similar clusters that would have formed $\gtrsim 5 \mathrm{Myr}$ ago, but that are still younger than the cluster disruption time-scale. However, no such clusters have been identified, suggesting that the formation of such clusters is relatively rare, possibly related to a coordinated burst of star formation triggered by the passage of a shock front, or that the cluster disruption scale is short. A number of isolated hot emission-line stars have also been detected in the central $\sim 40$ pc (see Cotera et al. 1999 and references therein).

Although NIR observations provide the most direct census of the stellar population, they are quite ineffective in highly obscured regions, such as the Galactic center GMCs. Therefore, largescale star-formation patterns in the Galactic center still remain largely unestablished. Searches for $\mathrm{OH}$ and $\mathrm{H}_{2} \mathrm{O}$ masers, which are also considered direct signposts of high-mass star formation resulted in relatively few detections (Güsten \& Downes 1983; Caswell et al. 1983; Morris 1989). The most recent published search for $\mathrm{H}_{2} \mathrm{O}$ masers in the inner $4^{\circ} \times 4^{\circ}$ region by Taylor et al. (1993) resulted in the detection of only 7 new maser sources (out of 97 IRAS selected sources observed), associated with star-forming regions that can be tentatively located at the Galactic center. Taylor et al. (1993) argue that the amount of molecular gas within $2^{\circ}$ of the Galactic center $\left(10^{7}-10^{8} \mathrm{M}_{\odot}\right)$ would normally contain a few hundred masers associated with protostars, $\mathrm{HH}$ objects, and T Tauri stars for a maser frequency comparable to that in the Galactic disk. While a large area unbiased survey for $\mathrm{H}_{2} \mathrm{O}$ masers has not yet been undertaken, it is quite clear that there are relatively few bright masers in the inner few hundred parsecs of the Galaxy. ${ }^{3}$ In a separate study Caswell (1996)

\footnotetext{
${ }^{3}$ The relatively low sensitivity of the maser searches carried out to date, owing to the large distance to the Galactic
} 
carried out a search for $6.668 \mathrm{GHz}$ methanol masers over a 2 square degree area, which resulted in the detection of 23 maser sites. Caswell argues that the space density of methanol masers in the Galactic center is similar to that in the spiral arms, implying that massive star-formation is neither enhanced nor suppressed in the Galactic center at the present time. However, these results are heavily influenced by the cluster of masers in the Sgr B2 complex, which accounts for nearly half of the maser sites detected.

In an effort to identify possible sites of future star formation (namely high-density GMC cores) in the Galactic center, Lis \& Carlstrom (1994) made an unbiased survey of the $800 \mu \mathrm{m}$ continuum emission over a $1.5^{\circ} \times 0.2^{\circ}$ region using the single pixel CSO bolometer. ${ }^{4}$ In this survey, in addition to the well studied sources like Sgr B2, Sgr C, and the 50 and $20 \mathrm{~km} \mathrm{~s}^{-1}$ clouds in Sgr A, a number of GMC cores were detected, forming a long $\left(\sim 0.25^{\circ}\right.$, or $\sim 40 \mathrm{pc}$ for a distance to the Galactic center of $8.5 \mathrm{kpc}$ ), clumpy ridge of dust emission (hereafter referred to as the "Ridge"), stretching from the radio Arc toward Sgr B2. No evidence for the presence of embedded FIR sources or compact HII regions associated with the brightest and most massive GMC core in the Ridge, GCM0.25+0.11, was found by Lis et al. (1994), and their VLA observations revealed the presence of only a weak $\mathrm{H}_{2} \mathrm{O}$ maser associated with this source, suggesting the presence of some ongoing low-mass star formation. The broad line widths $\left(\sim 30 \mathrm{~km} \mathrm{~s}^{-1} \mathrm{FWHM}\right)$, characteristic of Galactic center GMC, indicate that the clouds in the Ridge are indeed located within a few hundred parsecs of the Galactic center. In addition, as discussed by Lis \& Menten (1998; LM98), the velocity of the molecular emission in GCM0.25+0.01 is close to that of the molecular cloud associated with the HII region G0.18-0.04 (Serabyn \& Güsten 1991), which is believed to be interacting with the non-thermal filaments in the Galactic center radio Arc.

To better constrain the dust temperature and mass of the Ridge sources, we have obtained sensitive multi-wavelength images of the region with the Long Wavelength Spectrometer aboard the Infrared Space Observatory. Results for GCM0.25+0.11 have been previously presented by LM98. In the present paper we discuss continuum observations of the remaining ISO fields and refine our model for GCM0.25+0.01. We also present LWS observations of atomic fine structure lines of $\mathrm{CII}$ and OI and discuss their implications for the intensity of the diffuse interstellar radiation field in the region. In addition, millimeter-wave spectroscopic observations are used to independently constrain the physical conditions in the molecular gas. Finally, NIR imaging observations with the

center, may be partly responsible for the low maser detection rate. For example, a search by Mehringer et al. (1993), twice as sensitive as that of Taylor et al. (1993) ( $\sim 10 \mathrm{mJy}$ rms sensitivity compared to 16-22 mJy) resulted in the detection of 10 relatively weak $(50-430 \mathrm{mJy}) \mathrm{H}_{2} \mathrm{O}$ masers in a $7^{\prime} \times 7^{\prime}$ field in the $\mathrm{Sgr} \mathrm{B} 1$ region. As a comparison, Gaume et al. (1998) detected 36 masers with fluxes of 0.2-190 Jy at $15 \mathrm{mJy}$ sensitivity level in the Orion $\mathrm{S}$ region. Given the $\sim 20$ times larger distance to the Galactic center, many of these Orion masers would not be detectable in the existing Galactic center surveys.

${ }^{4}$ Much more sensitive, high-angular resolution submillimeter continuum surveys of the Galactic center region have been recently carried out by Dowell et al. (1999) using the SHARC bolometer camera at the CSO and by Pierce-Price et al. (2000) using the SCUBA array at the JCMT. 
Keck I telescope are used to address the nature of the $\mathrm{H}_{2} \mathrm{O}$ maser source in GCM0.25+0.01.

\section{Observations}

The ISO observations presented here consist of three $9 \times 9$ pixel maps with $50^{\prime \prime}$ spacing obtained with the LWS aboard of ISO. The instrument and calibration procedures are described by Clegg et al. (1996) and Swinyard et al. (1996). The data were taken during revolutions 497-498. At each position we obtained a grating wavelength-range spectrum (AOT LWS01) using all ten LWS detectors. The length of the spectrum was defined as $60.5-66.0 \mu \mathrm{m}$ for detector number three with a factor of 2 spectral oversampling and an integration time of 1.2 seconds per wavelength step. The approximate wavelength coverage for the ten LWS detectors is: $43.1-46.1 \mu \mathrm{m}, 50.4-56.1$ $\mu \mathrm{m}, 60.4-66.1 \mu \mathrm{m}, 70.1-75.7 \mu \mathrm{m}, 79.5-84.8 \mu \mathrm{m}, 91.0-102.5 \mu \mathrm{m}, 110.7-122.0 \mu \mathrm{m}, 130.5-141.8 \mu \mathrm{m}$, 149.7-160.7 $\mu \mathrm{m}$, and 167.6-178.1 $\mu \mathrm{m}$, respectively. The wavelength resolution is $\sim 0.11 \mu \mathrm{m}$ for detectors $1-5$, and $\sim 0.22 \mu \mathrm{m}$ for detectors $6-10$. The data were taken in total power mode so the maps are sensitive to the extended diffuse continuum emission. Each map was obtained with 81 independent pointings of the LWS. Initial data reduction (namely de-glitching and averaging) was performed using the ISO Spectral Analysis Package (ISAP). We used the "LSAN" files processed using pipeline 6.1 at the Rutherford Appleton Laboratories (RAL). In particular, flux calibration is part of the standard LWS pipeline processing.

Example LWS grating spectra are shown in LM98. Intensities (in $\mathrm{MJy} \mathrm{sr}^{-1}$ ) have been calculated using the measured beam sizes for the LWS detectors ( 61-82" FWHM). In addition to the thermal dust emission between $\sim 45-175 \mu \mathrm{m}$, several atomic fine structure lines and molecular lines can be distinguished in the spectra. These include the $51.81 \mu \mathrm{m}$ [OII] line, $63.18 \mu \mathrm{m}$ [OI] line, and the $157.7 \mu \mathrm{m}$ [CII] line, all seen in emission, as well as the $53.3 \mu \mathrm{m}$ and $119.3 \mu \mathrm{m}$ lines of $\mathrm{OH}$ seen in absorption. Intensities measured with long-wavelength detectors (detectors 6-10) are affected by fringing, with amplitude and period increasing with wavelength. Since the fringing does not have a significant effect on the dust temperatures and optical depth determination, we did not attempt to remove it during the data reduction.

Spectroscopic observations of millimeter lines of $\mathrm{CS}, \mathrm{C}^{34} \mathrm{~S},{ }^{13} \mathrm{CO}, \mathrm{H}_{2} \mathrm{CO}, \mathrm{SiO}, \mathrm{SO}$, and $\mathrm{CH}_{3} \mathrm{OH}$ (Table 1) were carried out in 1993-2000 using the facility receivers and spectrometers at the IRAM 30-meter telescope on Pico Veleta, Spain and the 10.4-meter Leighton telescope of the CSO on Mauna Kea, Hawaii. All the intensities presented here have been corrected for the corresponding main beam efficiencies given in Table 1.

Finally, J, H, K, and $\mathrm{Br} \alpha$ continuum $(4 \mu \mathrm{m})$ images of a $19^{\prime \prime} \times 19^{\prime \prime}$ field near the center of the GCM0.25+0.01 cloud were obtained in 1998 May at the Keck Observatory with the facility near-infrared camera, NIRC. The image scale is 0 . 15 per pixel and the seeing was $\sim 0$ " 4 . The data were dark-subtracted, flat-fielded and shift-added using standard IRAF subroutines. The "off" field was in the dark "hole" just above the Galactic center in latitude. The calibration was with 
respect to the UKIRT faint standard star \#34 (J, H, and K) and HD 201941 (Br $\alpha$ continuum).

\section{Discussion}

\subsection{Continuum Emission and Dust Temperature}

The far-infrared/submillimeter spectral energy distribution toward GCM0.25+0.11, the brightest submillimeter source in the Ridge, was discussed in detail in LM98. As expected, the distribution of long-wavelength $(173 \mu \mathrm{m})$ far-infrared emission throughout the whole region correlates well with that of the submillimeter continuum emission (Fig. 1). However, at MIR wavelengths $(\$ 70 \mu \mathrm{m})$ the submillimeter sources are either simply invisible, or in some cases show up in absorption against the general Galactic center background (Fig. 2). This has several interesting implications: (i) the GMC cores seen in the submillimeter continuum emission must be significantly colder than the diffuse dust component, (ii) they must have relatively high continuum opacities at MIR wavelengths (and consequently high $\mathrm{H}_{2}$ column density and mass), and (iii) these clouds seen in absorption must be located primarily in front of the warm dust responsible for the diffuse MIR emission. All the GMC cores in the Ridge appear as depressions in the $45 / 173 \mu \mathrm{m}$ color temperature map (Fig. 3 ). The upper limits to their dust temperature given by the color temperature are in the range $22-28 \mathrm{~K}$. However, the actual dust temperature for the bulk of the dust in these sources is even lower.

Owing to the broadband spectral coverage afforded by our ISO and CSO measurements (45$800 \mu \mathrm{m})$, we can constrain the dust temperature in the Ridge sources quite accurately. A two component gray-body model for GCM0.25+0.11 gives a mean dust temperature of $18 \mathrm{~K}$ (LM98; Table 2). A small amount of warmer dust $(\sim 35 \mathrm{~K})$ is required to accurately reproduce the observed SED, but this warmer dust contributes only $\lesssim 4 \%$ of the total optical depth and represents emission from the surface layer of the cloud externally heated by the diffuse interstellar radiation field. ${ }^{5}$ In addition, in order to reproduce the observed spectrum of this source a steep grain emissivity exponent $(\beta=2.8)$ is required. The continuum data presented here show that these results are typical for other sources in the region. Two component gray-body fits at several selected positions are shown in Figure 4 and the best fit parameters are listed in Table 2. The bulk of the dust in all the sources is quite cold $(15-22 \mathrm{~K})$ and the dust grains are characterized by steep values of $\beta=2.2-3.4$. The coldest dust is located in the northern part of the Sgr B1 cloud (Fig. 3, position ' $\mathrm{f}$ '). The mean dust temperature in the Ridge clouds derived here is lower than the global Galactic center average based on IRAS measurements ( $\sim 25-30 \mathrm{~K}$; Cox \& Laurijs 1989), as the IRAS average represents a combination of cold, dense GMCs and a warmer diffuse dust component.

\footnotetext{
${ }^{3}$ While the FIR data are taken in total-power mode, the submillimeter data are beam-switched observations and the low-level extended emission is filtered out in the maps. Therefore, a constant offset corresponding to the lowest flux pixel was subtracted from the observed flux densities at FIR wavelengths before fitting the SEDs. This background removal technique is much simpler than that used by LM98 for GCM0.25+0.01, but it results in essentially the same best fit temperature of the cold component ( $17 \mathrm{~K}$ vs. $18 \mathrm{~K}$ ).
} 
The low dust temperature in the Ridge is a natural consequence of the absence of embedded heating sources - the dust is primarily heated externally by the diffuse interstellar radiation field. The steep value of $\beta$ has been interpreted by LM98 as evidence for the presence of dust grains covered with thick ice mantles (see the discussion of $\beta$ in Lis et al. 1998); the molecules are expected to stick to the dust grains at the high densities and low temperatures characteristic of these sources. As discussed in LM98, the steep values of $\beta$ derived from the gray-body fits are not an artifact of the calibration mismatch between the FIR and submillimeter data, as $\beta=2.6$ is derived for GCM0.25+0.01 from a fit to the ISO data alone. Given the calibration uncertainties of the continuum data $(\sim 25 \%)$, the maximum $(3 \sigma)$ uncertainty of $\beta$ is \pm 0.8 (see Fig. 4 of LM89).

\subsection{Continuum Radiative Transfer Models}

As discussed above, the low mean dust temperature derived from the simple gray-body fits to the observed SEDs is consistent with external heating of the dust in the dense GMCs cores in the Ridge. To test this assertion more quantitatively, we have modeled the observed SED of GCM $0.25+0.11$ using the radiative transfer code of Egan et al. (1988) with the objective of deriving the external radiation field intensity and the internal source luminosity required to reproduce the observed spectrum. Grain parameters are those derived by LM98, namely the grain emissivity exponent $\beta=2.8$ at FIR/submillimeter wavelengths $(\varsigma 50 \mu \mathrm{m})$ and the $350 \mu \mathrm{m}$ grain emissivity $Q_{350}=1 \times 10^{-4}$. At wavelengths shorter than $50 \mu \mathrm{m}$ we assume a shallower grain emissivity exponent $\beta=1$. The external radiation field intensity is based on the tabulation of Mathis et al. (1983). The FIR part of the radiation field in the vicinity of GCM0.25+0.01 is measured directly with ISO (see Fig. 3 of LM98) and it corresponds to a factor of $\sim 120$ enhancement compared to the solar neighborhood values given by Mathis et al. (1983). The more recent COBE measurements, indicate that the tabulation of Mathis et al. underestimates the FIR radiation field intensity by a factor of $\sim 3$ (Black 1994). The FIR radiation field enhancement in the vicinity of GCM0.25+0.01 is thus a factor of $\sim 40$ compared to the COBE measurements. The radiation field intensity at shorter wavelengths is not measured directly, and we use an arbitrary enhancement factor, $G_{o}$, at UV-NIR wavelengths $(\lambda \lesssim 10 \mu \mathrm{m})$ as an adjustable model parameter. To minimize the number of free parameters, we assume a spherically symmetric model cloud with an $r^{-2}$ density distribution. This is a rather crude approximation for GCM $0.25+0.01$, but sufficient for $G_{o}$ determination. The outer radius of the model cloud is $150^{\prime \prime}$, corresponding to the mean extent of the submillimeter continuum emission. The UV radiation field enhancement factor, $G_{o}$, the internal luminosity, $L_{o}$, and the dust optical depth at the reference wavelength of $350 \mu \mathrm{m}$ are then the only adjustable parameters in the models.

The minimum UV field enhancement consistent with our FIR measurements of the diffuse dust

component in the vicinity of GCM0.25+0.01 and the COBE spectrum is $G_{o}=40$. The solid line in Figure 5 (top panel) shows a fit to the FIR/submillimeter part of the spectrum $(\lambda \gtrsim 100 \mu \mathrm{m})$, assuming $G_{o}=40$ with $L_{o}$ as an adjustable parameter; the best fit corresponds to an internal 
luminosity $L_{o}=2.5 \times 10^{5} \mathrm{~L}_{\odot}$. The figure demonstrates the failure of internally heated models to reproduce the MIR part of the spectrum $(\lambda \lesssim 100 \mu \mathrm{m})$; the SED in internally heated models is simply too narrow compared to the observed SED in GCM0.25+0.01. Externally heated models produce a much broader SED (see the dashed line in Fig. 5, top panel, corresponding to $G_{o}=500, L_{o}=0$ ), however they underestimate the observed emission near the peak of the spectrum $(\sim 100 \mu \mathrm{m})$. Since the external UV field enhancement factor and the internal luminosity affect different parts of the spectrum, both parameters can be constrained independently by the models. The solid line in Figure 5 (middle panel) shows our best fit model including both external and internal heating, which corresponds to $G_{o}=500$ and $L_{o}=1.9 \times 10^{5} \mathrm{~L}_{\odot}$. The two dotted lines in this panel correspond to models with $L_{o}=1.0 \times 10^{5}$ and $2.7 \times 10^{5} \mathrm{~L}_{\odot}$; these two values bracket the internal source luminosity, which given a $25 \%$ calibration uncertainty is still consistent with the FIR and submillimeter data. The effect of $G_{o}$ on the model SEDs is shown in Figure 5 (bottom panel). The solid line shows again the best fit model, corresponding to $G_{o}=500$ and $L_{o}=1.9 \times 10^{5} \mathrm{~L}_{\odot}$. The two dotted lines show models corresponding to $G_{o}=400$ and 600 , which are still consistent with the data given the calibration uncertainties.

Increasing the outer cloud radius by $50 \%$ has no effect on the best fit value of $G_{o}$ and lowers $L_{o}$ by $\sim 5 \%$. Using a shallower density law of $r^{-1}$ instead of $r^{-2}$ increases the best fit $G_{o}$ by $\sim 20 \%$ and lowers $L_{o}$ by $\sim 10 \%$. In order to obtain a satisfactory fit in the $50-100 \mu \mathrm{m}$ wavelength range for the $r^{-1}$ density profile we have to lower $\beta$ from 2.8 to 2.4 . We thus conclude that the best fit values of the UV field enhancement factor and the internal source luminosity are only weakly dependent on the detailed model cloud parameters and are well constrained by the models. Our best fit estimate of $G_{o}$ is $500 \pm 100$ and the best fit $L_{o} \simeq 2 \times 10^{5} \mathrm{~L}_{\odot}$, with a factor of $\sim 2$ uncertainty. The upper limit for the internal luminosity of $2.7 \times 10^{5} \mathrm{~L}_{\odot}$ corresponds to $\sim 4$ B0 ZAMS stars (Cox 2000).

\subsection{Atomic Fine Structure Lines}

In addition to the continuum SEDs, our LWS grating spectra cover the $63 \mu \mathrm{m}$ fine structure line of OI and the $158 \mu \mathrm{m}$ line of CIr. Observations of these lines, together with the FIR continuum emission, allow independent determination of the physical conditions in the diffuse ISM in the Galactic center region, namely the gas density and the ISRF intensity, through PDR modeling.

Wolfire et al. (1990) calculated a grid of PDR models for a range of hydrogen densities $\left(n_{\mathrm{H}}\right)$ and UV field intensities $\left(G_{o}\right)$ and showed that the CII/OI and (CII+OI+SiIl)/FIR intensity ratio can be used to constrain independently the values of $n_{\mathrm{H}}$ and $G_{o}$ (see Fig. 6). ${ }^{6}$ The gray points in Figure 6 correspond to several lines of sight within our ISO maps, away from the dense cores, which cover a wide range of the $45 / 173 \mu \mathrm{m}$ color temperatures. The points cluster around

\footnotetext{
${ }^{6}$ As discussed by Simpson et al. (1997), the intensity of the $35 \mu \mathrm{m}$ Sill line which is included in the calculations of Wolfire et al. (1990) can be neglected, since Si is highly depleted in their models.
} 
$G_{o}=1000$ and $n_{\mathrm{H}}=1000 \mathrm{~cm}^{-3}$. The derived values of $n_{\mathrm{H}}$ and $G_{o}$ are uncertain to within a factor of $\sim 2$ owing to uncertainties in the optical depth of the fine structure lines. The value of $G_{o}$ derived from the fine structure line observations thus agree with that obtained in $\S 3.2$ from the continuum radiative transfer modeling. We therefore have a consistent model of the continuum emission from GCM0.25+0.01, which is primarily externally heated by the diffuse Galactic center radiation field with the intensity $\sim 600$ times the standard ISRF, with only limited internal heating $\left(L_{o} \simeq 1.5 \times 10^{5} \mathrm{~L}_{\odot}\right)$. Other GMC cores in the Ridge have FIR fluxes and temperatures (and thus column densities, volume densities, and masses) similar to those of GMC0.25+0.01 (Table 2). These modeling results can thus be considered as representative for all the sources in the Ridge.

\subsection{Molecular Observations and LVG Modeling}

The continuum observations and modeling presented above support the conclusion that dust grains in the GMC cores in the region between the radio Arc and Sgr B2 are externally heated by the diffuse interstellar radiation field and have mean dust temperatures of $\sim 15-22 \mathrm{~K}$. Since the mean densities characteristic of these objects are $\sim 10^{5} \mathrm{~cm}^{-3}$ (see below) the gas and dust are expected to be relatively well coupled (Goldsmith \& Langer 1978; Doty \& Neufeld 1997). Nevertheless, it is important to determine independently the physical conditions in the gas from observations of carefully chosen molecular tracers. In order to study the molecular gas properties, we carried out observations of selected transitions of $\mathrm{H}_{2} \mathrm{CO}, \mathrm{CS}, \mathrm{C}^{34} \mathrm{~S}, \mathrm{SiO}, \mathrm{SO}$, and $\mathrm{CH}_{3} \mathrm{OH}$ using the IRAM 30-meter telescope and the CSO (see Table 1).

The dependence of $\mathrm{H}_{2} \mathrm{CO}$ line ratios on the gas density and kinetic temperature was studied extensively by Mangum \& Wootten (1993). $\mathrm{H}_{2} \mathrm{CO}$ is a slightly asymmetric rotor, therefore transitions involving the same $J$-levels, which come from different $K$-levels are closely spaced in frequency and can often be observed in a single receiver frequency setting. By measuring the intensities of two $K$-components from the same $\Delta J=1$ transition, one can obtain a measure of the kinetic temperature, which is unaffected by relative pointing uncertainties, beam-size differences, and calibration uncertainties. In particular, the intensity ratio of the $\left(3_{03}-2_{02}\right)$ and $\left(3_{22}-2_{21}\right)$ transitions of para- $\mathrm{H}_{2} \mathrm{CO}$ (at 218.222192 and $218.475632 \mathrm{GHz}$, respectively) is a good temperature probe for $T_{K} \lesssim 50 \mathrm{~K}$ and $N\left(\mathrm{H}_{2} \mathrm{CO}\right) / \delta v \lesssim 10^{13.5} \mathrm{~cm}^{-2}\left(\mathrm{~km} \mathrm{~s}^{-1}\right)^{-1}$ (Mangum \& Wootten 1993).

We have observed the $\mathrm{H}_{2} \mathrm{CO}\left(3_{03}-2_{02}\right)$ and $\left(3_{22}-2_{21}\right)$ lines at four positions in the Ridge. Figure 7 shows the observed spectra and the corresponding line intensities are given in Table 3. The $\left(3_{03}-2_{02}\right) /\left(3_{22}-2_{21}\right)$ line intensity ratio varies between 3 and 4 with a fractional uncertainty of $5-15 \%(1 \sigma)$ at any given position. Inspection of Figure 13 of Mangum \& Wootten (1993) gives the

corresponding gas temperatures of $60-90 \mathrm{~K}$ for a gas density of $\sim 10^{5} \mathrm{~cm}^{-3}$ (see below), assuming optically thin emission. The allowed temperature range depends only weakly on the gas density in the range $\sim 10^{4.5}-10^{5.5} \mathrm{~cm}^{-3}$ and shifts toward higher values with increasing density, to $\sim 80-180 \mathrm{~K}$ for a density of $10^{6} \mathrm{~cm}^{-3}$. The line ratio of $3.13 \pm 0.17$ observed in GCM0.25+0.01 corresponds to the gas temperature of $80(+40,-15) \mathrm{K}(3 \sigma$ uncertainties). In fact, gas temperatures at all four 
positions are above $50 \mathrm{~K}$ at $3 \sigma$ level, significantly higher than the dust temperatures derived above.

In a PDR, collisions with dust grains are the dominant heating source for the gas in the cloud interior ( 28 magnitudes of visual extinction; see Fig. 1 of Hollenbach et al. 1991; their "standard model" corresponds to the UV enhancement factor of 1000 and $\mathrm{H}$ nuclei density of $1000 \mathrm{~cm}^{-3}$, comparable to within a factor of a few to those in the outer layers of the Ridge clouds). Therefore the dust temperature in the cloud interior is always higher than the gas temperature (see Fig. 3 of Hollenbach et al.). The fact that the gas temperature in GCM0.25+0.01 exceeds the dust temperature thus indicates that the reprocessed UV radiation is not the dominant heating source for the molecular gas.

Carey et al. (1998) observed several $\mathrm{H}_{2} \mathrm{CO}$ transitions in a sample of sources detected in absorption at 8-25 $\mu \mathrm{m}$ by the MSX satellite. They derive significantly lower gas temperatures ( $\$$ $20 \mathrm{~K}$ ) for all sources in their sample, which also includes GCM0.25+0.01. When taken at face value, our observations of the $218 \mathrm{GHz} \mathrm{H} \mathrm{H}_{2} \mathrm{CO}$ lines seem to contradict the results of Carey et al. (1998). However, as discussed by Carey et al., a range of possible solutions ranging from low temperature $(<15 \mathrm{~K})$ and high density $\left(>3 \times 10^{5} \mathrm{~cm}^{-3}\right)$ to a higher temperature $(>40 \mathrm{~K})$ and lower density $\left(\sim 10^{5} \mathrm{~cm}^{-3}\right)$ is consistent with their observed line ratios. While Carey et al. favor the low-temperature, high-density solution for GCM0.25+0.01, our observations are more consistent with their high-temperature, low-density solution, which has a comparable $\chi^{2}$ value (see Fig. 4 of Carey et al.)

Our temperature determination could be in error if the $\mathrm{H}_{2} \mathrm{CO}$ lines are optically thick, and so we searched for the corresponding $\mathrm{H}_{2}^{13} \mathrm{CO}$ transitions at $213 \mathrm{GHz}$ at position ' $\mathrm{a}$ '. No emission was detected with a $3 \sigma$ lower limit for the $\mathrm{H}_{2} \mathrm{CO} / \mathrm{H}_{2}^{13} \mathrm{CO}$ ratio of 36 . Assuming a ${ }^{12} \mathrm{C} /{ }^{13} \mathrm{C}$ ratio of 24 in the Galactic center clouds (Langer \& Penzias 1990), this implies that the $\mathrm{H}_{2} \mathrm{CO}$ emission at the position we observed is optically thin and our temperature determination should not be adversely affected by optical depth effects.

With a single pair of $\mathrm{H}_{2} \mathrm{CO}$ lines observed, we cannot rule out the possibility that several components with varying temperatures are present along the line of sight. In fact, $\mathrm{NH}_{3}$ data of Hüttemeister et al. (1993) show that two gas components with different temperatures are often present in Galactic center cores without high-mass star formation: a cool component $(\sim 20-30 \mathrm{~K})$ and a warm component $(z 120 \mathrm{~K})$. A $\sim 75 \mathrm{~K}$ kinetic temperature often derived in excitation studies of the Galactic center sources may simply be a weighted average of the two components (Hüttemeister et al. 1998). This may also be the case for GCM0.25+0.01, but it does not affect the conclusion that considerable fraction of the relatively dense molecular gas has a kinetic temperature far above the dust temperature in the Ridge sources. This result is consistent with the ammonia measurements of Hüttemeister et al. (1993), which suggest that a warm gas component is widespread in the Galactic center region.

In order to determine the gas density in GCM0.25+0.01 we observed the (2-1), (3-2), and (5-4) transitions of the $\mathrm{CS}$ and $\mathrm{C}^{34} \mathrm{~S}$ molecules. The spectra are shown in Figure 8 and the 
integrated intensities corrected for the corresponding main beam efficiencies are given in Table 4 . Results of LVG modeling of the data are presented in Figure 9. The isotopic CS data alone cannot independently constrain both the gas density and kinetic temperature. A range of models placed along a line running diagonally across the plot are statistically consistent with the data. Based on a formal $\chi^{2}$ fit, the $\mathrm{C}^{34} \mathrm{~S}$ data favor a model with a kinetic temperature of $\sim 50 \mathrm{~K}$ and $\mathrm{H}_{2}$ density of $\sim 1.4 \times 10^{5} \mathrm{~cm}^{-3}$, while the CS data imply an even higher temperature of $\sim 150 \mathrm{~K}$ and a density of $\sim 7 \times 10^{4} \mathrm{~cm}^{-3}$. In both cases, the best fit kinetic temperature is significantly higher than that derived by Carey et al. (1998) from their $\mathrm{H}_{2} \mathrm{CO}$ measurement $(<20 \mathrm{~K})$. Using the kinetic temperature derived from our $\mathrm{H}_{2} \mathrm{CO}$ observations, along with the isotopic $\mathrm{CS}$ data we can better constrain the gas density. The resulting best fit $\mathrm{H}_{2}$ volume density assuming an $80 \mathrm{~K}$ kinetic temperature is $n_{\mathrm{H}_{2}} \simeq 1 \times 10^{5} \mathrm{~cm}^{-3}$, with an uncertainty of $\lesssim 40 \%$.

\subsection{Gas Heating}

The apparent discrepancy between our derived gas and dust temperatures in the Ridge indicates that gas heating in this region is dominated by processes other than UV radiation. In our best fit dust model including both internal and external heating, the dust temperature is above $80 \mathrm{~K}$ only in the central $\sim 2^{\prime \prime}$ region, which contains $\sim 1.4 \%$ of the total molecular mass. The presence of multiple heating sources and a clumpy structure of the cloud core would tend to increase the size of the warm region. Nevertheless, it would not be sufficient to explain the large amount of warm $\mathrm{H}_{2} \mathrm{CO}$ observed. PDR models predict the presence of a thin layer of warm $(\$ 100 \mathrm{~K}$ ) gas near the surface of the cloud (at $\lesssim 3$ magnitudes of visual extinction; see Fig. 3 of Hollenbach et al. 1991). However, the gas in this region is atomic rather than molecular (see Fig. 4b of Hollenbach et al.) and $\mathrm{H}_{2} \mathrm{CO}$ is not expected to be present.

LM98 suggested that the complicated gas kinematics in GCM0.25+0.01 may be indicative of a collision between two separate clouds. Velocity channel maps of the ${ }^{13} \mathrm{CO}(2-1)$ and $\mathrm{CS}(3-2)$ at $11^{\prime \prime}$ and $18^{\prime \prime}$ angular resolution are shown in Figures 10 and 11 , respectively. The northern, relatively extended cloud is best seen in the $20 \mathrm{~km} \mathrm{~s}^{-1}$ panel. The southern cloud runs diagonally from NE to SW in the lower half of the $40 \mathrm{~km} \mathrm{~s}^{-1}$ panel and exhibits a large velocity gradient at the SW tip. While the ${ }^{13} \mathrm{CO}$ emission is relatively extended, the CS emission tracing high-density gas is more clumpy and significantly brighter in the southern cloud.

To search for evidence of shocks associated with the collision of the two clouds we have observed the $\mathrm{SiO}(5-4)$ emission at 12 positions forming a cross centered on the CS (3-2) peak. The observed spectra are shown in Figure 12. The SiO emission is strong and widespread in GCM0.25+0.01. The $\mathrm{SiO}$ peak coincides with the $\mathrm{CS}$ (3-2) peak and is not associated with the embedded $\mathrm{H}_{2} \mathrm{O}$ maser source (Lis et al. 1994), which is located $\sim 80^{\prime \prime}$ to the north. The CS emission is somewhat more extended than the $\mathrm{SiO}$ emission, possibly owing to the higher optical depth.

The distribution and excitation of the $\mathrm{SiO}$ emission in the Galactic center was studied by 
Martín-Pintado et al. (1997; only the southernmost part of the Ridge was covered in that study with sparse spatial coverage) and Hüttemeister et al. (1998). In contrast to the spatial distribution of other high-density tracers, such as $\mathrm{CS}$, the $\mathrm{SiO}$ emission is very fragmented on a large scale and is associated only with selected molecular clouds. As discussed by Martín-Pintado et al. (1997), the particular chemistry of these clouds is probably related to large-scale fast shocks occurring in the Galactic center. $\mathrm{SiO}$ is an unambiguous tracer of high-temperature and shock chemistry. In Galactic disk clouds the $\mathrm{SiO}$ emission is associated with energetic molecular outflows powered by young stars. $\mathrm{Si}$ is normally highly depleted and $\mathrm{SiO}$ is present only in the regions where shock disruption of grains releases Si to the gas phase (Martín-Pintado et al. 1992). The Galactic center $\mathrm{SiO}$ seems to be associated with warm gas and high-temperature chemistry and could explain the $\mathrm{SiO}$ abundances if $\mathrm{Si}$ is less depleted compared to the disk (Martín-Pintado et al. 1997). Fast shocks in the $\mathrm{SiO}$ clouds are likely of different origin for different $\mathrm{SiO}$ complexes. For example, in the Sgr A complex they can be produced by the expansion of the Sgr A East SNR and in the radio Arc area by the interaction of molecular material with the non-thermal filaments. However, the absence of associated radio continuum emission makes this explanation unlikely for the Ridge clouds. While a collision of two clouds may be responsible for the enhanced SiO emission in GCM0.25+0.01, the other sources in the region with strong SiO emission (e.g. Sgr B1) appear to have a much simpler velocity field.

The correlation between the SiO morphology and the spatial distribution of the Fe $6.4 \mathrm{keV}$ line in the Galactic center (Martín-Pintado et al. 2000) may offer an alternative explanation for the strong $\mathrm{SiO}$ emission observed in Galactic center clouds. While it is possible that in some regions the sources driving the shocks responsible for grain destructions also generate hard X-rays which excite the Fe $6.4 \mathrm{keV}$ line (e.g. the expansion of $\mathrm{Sgr} A$ East into the $50 \mathrm{~km} \mathrm{~s}^{-1}$ cloud), other Galactic center clouds, including Sgr B2, could simply be X-ray reflection nebulae illuminated by a time variable source of hard X-ray emission located outside the neutral cool material (Koyama et al. 1996). GCM0.25+0.01 and other Ridge clouds can also be examples of X-ray irradiated molecular clouds, which contain regions of high temperature with enhanced Si abundance in the gas phase. However, emission from other shock tracers, such as $\mathrm{SO}$ and $\mathrm{CH}_{3} \mathrm{OH}$ (see Bachiller 1996 and references therein), is also strong and widespread in the Ridge area (see Figs. 7 and 12). A more complete, fully sampled map of $\mathrm{SiO}$ emission and other shock tracers in the Ridge area is required to better understand the correlations of $\mathrm{SiO}$ emission with the high-column density GMC cores seen in the submillimeter continuum emission.

\subsection{NIR Imaging}

Given the high column density of the GMC cores in the Ridge $\left(\sim 3 \times 10^{24} \mathrm{~cm}^{-2}\right.$ in GCM0.25+0.01 in a $30^{\prime \prime}$ beam; Table 2), corresponding to a few hundred magnitudes of K-band extinction, a direct detection of any embedded sources via NIR imaging is simply not feasible. However, NIR obser-

vations could help to determine the nature of the $\mathrm{H}_{2} \mathrm{O}$ maser source in GCM0.25+0.01. If the 
$\mathrm{H}_{2} \mathrm{O}$ maser is associated with an evolved star located in front of the dust core, it would be easily detectable at IR wavelengths (an evolved star located behind the core would still be extincted by the dust and therefore not detectable in the NIR). To search for the presence of IR sources in the vicinity of the $\mathrm{H}_{2} \mathrm{O}$ maser, we imaged a $\sim 19 \times 19^{\prime \prime}$ region at the $\mathrm{J}, \mathrm{H}, \mathrm{K}$, and $\mathrm{Br} \alpha$ continuum wavelengths using the NIRC instrument at the Keck I telescope.

Figure 13 shows the NIR images. A group of three strong K-band sources are detected in the vicinity of the $\mathrm{H}_{2} \mathrm{O}$ maser. Their magnitudes and IR colors are given in Table 5 . The three stars are located slightly to the left of the reddening line in the $\mathrm{J}-\mathrm{H} / \mathrm{H}-\mathrm{K}$ color-color diagram (see Fig. 6 of Blum et al. 1996). This indicates that they are late-type giants rather than embedded YSOs. Assuming IR colors of an M-giant $(\mathrm{J}-\mathrm{H}=0.7$ and $\mathrm{H}-\mathrm{K}=0.3)$, we derive $E_{H-K}=$ $(H-K)-(H-K)_{o}=1.2$ for the brightest star. The corresponding value of the $\mathrm{K}$-band extinction is $A_{K}=X E_{H-K}$ (e.g. Figer et al. 1998). With $\mathrm{X}=1.8$ (Rieke et al. 1989), we derive $A_{K}=$ 2.1 , comparable to the typical values derived for the members of the central $r^{-2}$ cluster. The corresponding visual extinction is $A_{V}=A_{K} / 0.112=24$ (Rieke et al. 1989). The remaining two stars have similar IR colors and thus extinction values. The absolute K-band magnitude of the brightest star is 6.7 , toward the high end of the Galactic center K-band luminosity function (see Fig. 8 of Blum et al. 1996).

The registration of the IR images is uncertain, since the star guider was not operational during our observations. To best determine the registration, we determined the telescope pointing using the familiar stellar pattern in the vicinity of the Sgr $A^{*}$ source at the dynamical center of the Milky Way. We then offset the telescope to the position of the $\mathrm{H}_{2} \mathrm{O}$ maser in GCM0.25+0.01. The square box in the lower-right corner shows the expected position of the maser source, which is $\sim 7.4^{\prime \prime}$ west and $\sim 8.6^{\prime \prime}$ south of the brightest IR source. Given the large position offset, we conclude that the three evolved stars detected in our IR images are most likely unrelated to the $\mathrm{H}_{2} \mathrm{O}$ maser source. No candidate IR source associated with the maser has been detected in our images. ${ }^{7}$ This is consistent with the conclusion of Lis et al. (1994) that the maser is most like not associated with an evolved star, but instead with a deeply embedded intermediate or low-mass YSO.

\subsection{Star Formation in the Ridge}

As discussed in $\S 3.2$, the observed SED of GCM0.25+0.01 is consistent with the presence of an internal heating source equivalent to $\$ 4$ main sequence B0 stars. It is informative to consider how many such stars can be expected to form in this cloud, given its total molecular mass $\left(\$ 1 \times 10^{6} \mathrm{M}_{\odot}\right.$; LM98, an upper limit to the virial mass based on CO data). The stellar initial mass function is defined as $n(M) d M=n_{o} M^{\Gamma-1} d M$, where $\Gamma=-1.35$ is the Salpeter slope (e.g. Elmegreen 1999).

\footnotetext{
${ }^{7}$ Although the maser is located very close to the edge of the images (see the square box in Fig. 13), the expected maser position is completely covered at the $H$ and $K$ wavelengths.
} 
Given the upper limit of $1 \times 10^{6} \mathrm{M}_{\odot}$ for the molecular mass of GCM0.25+0.01, and assuming a star formation efficiency of $1 \%$ and a Salpeter IMF with the lower and upper mass cutoffs of 0.1 and $60 \mathrm{M}_{\odot}$, respectively, the number of stars of spectral type $\mathrm{B} 0$ or earlier (with stellar masses greater than $17.5 \mathrm{M}_{\odot}$ ) expected to form over the lifetime of this cloud is $\sim 20$. This is an order of magnitude higher than the maximum number allowed by our continuum models. The expected number of early type stars increases significantly for a shallower IMF, such as that derived by Figer et al. (1999) for the Arches and Quintuplet clusters ( $\sim 120 \mathrm{~B} 0$ stars for $\Gamma=-0.65)$.

On the other hand, the radio continuum observations of Lis et al. (1994) impose an even more stringent limit on the number of massive stars associated with GCM0.25+0.01. The 3 $\sigma$ upper limit of $1.1 \mathrm{mJy}$ in a $2.6^{\prime \prime}$ beam for the $8.4 \mathrm{GHz}$ continuum flux density within the boundaries of the submillimeter continuum emission gives a Lyman continuum luminosity of $\sim 8 \times 10^{45} \mathrm{~s}^{-1}$, which corresponds to a single B0.5-B1 ZAMS star (Panagia 1973). The presence of stars of spectral types earlier than B0.5 in GCM0.25+0.01 is thus excluded by the $8.4 \mathrm{GHz}$ observations. We thus conclude that either (a) the current star formation efficiency in the Ridge must be very low, on the order of $0.1 \%$ or lower, or alternatively (b) the upper mass cutoff of the IMF for any stars already formed must be $\lesssim 10 \mathrm{M}_{\odot}$ to explain the FIR and radio continuum data. Both of these conclusions are consistent with a quiescent cloud, still largely in a pre-collapse phase.

\section{Summary}

The observations presented here firmly establish that the GMCs in the Ridge, located between the Galactic center radio Arc and the Sgr B2 molecular cloud, are different from the well studied starforming Galactic center clouds, such as Sgr B2, Sgr C, Sgr D, or the $50 \mathrm{~km} \mathrm{~s}^{-1}$ cloud in Sgr A. Our FIR continuum and fine structure line observations imply low dust temperatures $(15-22 \mathrm{~K})$ in all the sources we studied, consistent with external heating by the diffuse interstellar radiation field with an enhancement factor $G_{o} \simeq 500-1000$. We find no evidence for the presence of luminous internal heating sources and thus for ongoing high-mass star formation associated with these GMCs, in spite of their large molecular masses.

The kinetic temperatures in the Ridge sources appear significantly higher than the dust temperatures. We suggest that shocks associated with cloud collisions may be the dominant heating source for the gas in this region. In fact, molecular observations of GCM0.25+0.01 show complicated gas kinematics, possibly indicating an early stage of a cloud-cloud collisions. However, the remaining sources show a simpler velocity field.

The integrated $350 \mu \mathrm{m}$ flux density in a $\sim 50 \times 70 \mathrm{pc}$ region including the Ridge is $\sim 100 \mathrm{kJy}$ (this only includes pixels with $350 \mu \mathrm{m}$ fluxes greater than $50 \mathrm{Jy}$ in a $15^{\prime \prime}$ beam, corresponding to a $8 \sigma$ detection; the cutoff is introduced to separate the emission from the dense GMC cores from that of the low-density extended component that contributes additional $50 \mathrm{kJy}$ to the integrated flux density in the region). Assuming a dust temperature of $20 \mathrm{~K}$, the combined molecular mass 
of all the Ridge sources is $\sim 1 \times 10^{7} \mathrm{M}_{\odot}$, comparable to that of Sgr B2, the most massive GMC in the Galactic center region ( $L$ is \& Goldsmith 1989). These clouds are thus a significant component of the Galactic center ISM. Owing to the time constraints, we have been able to study in detail only a limited sample of Galactic center GMCs with ISO. However, the shorter wavelength MSX continuum observations of Egan et al. (1998) indicate that a large number of presumably similar, cold dust sources are present in the Galactic center region and throughout the Galaxy. We thus suggest that quiescent clouds, such as those that we have studied here actually represent "typical" pre-collapse Galactic center GMCs, as opposed to the well known, bright star-forming clouds like Sgr B2, Sgr C, Sgr D, or the $50 \mathrm{~km} \mathrm{~s}^{-1}$ cloud in Sgr A. The latter sources were all identified because of their strong FIR and/or radio continuum emission, which are natural tracers of high-mass star formation. Therefore they may nearly represent that subset of the Galactic center cloud population, in which energetic activity (shocks, compression) and prolific high-mass star formation are already present. The presence of shock activity in GCM0.25+0.01, as evidenced by the widespread $\mathrm{SiO}$ emission, indicates that star formation may be "turning on" at least in this cloud.

This research has been supported by NSF grant AST-9980846 to the Caltech Submillimeter Observatory and by NASA under an ISO Block Grant to the California Institute of Technology. The NIR data presented here were obtained at the W.M. Keck Observatory, which is operated as a scientific partnership between The California Institute of Technology, the University of California, and NASA. The observatory was made possible by the generous financial support of the W.M. Keck Foundation. The ISO Spectral Analysis Package (ISAP) is a joint development by the LWS and SWS Instrument Teams and Data Centers. Contributing institutes are CESR, IAS, IPAC, MPE, RAL and SRON. We thank the referee, N. Evans, for a thorough and helpful report, M. Morris for helpful comments, and P. Schilke for providing us with a LVG code for CS.

\section{REFERENCES}

Bachiller, R. 1996, ARAA, 34, 111

Bally, J., Stark, A.A., \& Wilson, R.W. 1988, ApJ, 324, 223

Black, J.H. 1994, in The First Symposium on the Infrared Cirrus and Diffuse Interstellar Clouds, eds. R. Curti \& W. Latter, (ASP Conf. Series), 355

Blum, R.D., Sellgren, K., \& DePoy, D.L. 1996, ApJ, 470, 864

Carey, S.J., Clark, F.O., Egan, M.P., Price, S.D., Shipman, R.F., \& Kuchar, T.A. 1998, ApJ, 508, 721

Caswell, J.L. 1996, MNRAS, 283, 606

Caswell, J.L., Batchelor, R.A., Forster, J.R., \& Wellington, K.J. 1983, AuJPh, 36, 401 
Clegg, P.E. et al. 1996, A\&A, 315, L38

Cotera, A.S., Simpson, J.P., Erickson, E.F., \& Colgan, S.W.J. 1999, ApJ, 510, 747

Cox, A.N., ed. 2000, Allen's Astrophysical Quantities, (Springer-Verlag: New York)

Cox, P., \& Laurijs, R. 1989, in The Center of the Galaxy, ed. M. Morris, (Dordrecht: Kluwer), 121

Doty, S.D. \& Neufeld, D.A. 1997, ApJ, 489, 122

Dowell, C.D., Lis, D.C., Serabyn, E., Gardner, M., Kovacs, A., \& Yamashita, S. 1999, in The Central Parsecs of the Galaxy, ed. H. Falcke et al. (ASP Conf. Series), 453

Egan, M.P., Leung, C.M., \& Spagna, G.F. 1988, Computer Phys. Comm., 48, 271

Egan, M.P., Shipman, R.F., Price, S.D., Carey, S.J., \& Clark, F.O. 1998, ApJ494, L199

Elmegreen, B.G. 1999, ApJ, 515, 323

Figer, D.F., Kim, S.S., Morris, M., Serabyn, E., Rich, R.M., \& McLean, I.S. 1999b, ApJ, 525, 750

Gaume, R.A., Wilson, T.L., Vrba, F.J., Johnston, K.J., \& Schmidt-Burgk, J. 1998, ApJ, 493, 940

Goldsmith, P.F., Bergin, E.A., \& Lis, D.C. 1997, ApJ, 491, 615

Goldsmith, P.F., \& Langer, W.D. 1978, ApJ, 222, 881

Güsten, R. \& Downes, D. 1983, A\&A, 117, 343

Hildebrand, R.H. 1983, QJRAS, 24, 267

Hollenbach, D.J., Takahashi, T., \& Tielens, A.G.G.M. 1991, ApJ, 377, 192

Hüttemeister, S., Dahmen, G., Mausberger, R., Henkel, C., Wilson, T.L., \& Martín-Pintado, J. 1998, A\&A, 334, 646

Hüttemeister, S., Wilson, T.L., Bania, T.M., \& Martín-Pintado, J. 1993, A\&A, 280, 25

Koyama, K., Maeda, Y., Sonobe, T., Takeshima, T., Tanaka, Y., \& Yamauchi, S. 1996, PASJ, 48, 249

Langer, W.D., \& Penzias, A.A. 1990, ApJ, 357, 477

Lis, D.C., \& Carlstrom, J.E. 1994, ApJ, 424, 189

Lis, D.C., \& Goldsmith, P.F. 1989, ApJ, 337, 704

Lis, D.C., \& Menten, K.M. 1998, ApJ, 507, 794 (LM98)

Lis, D.C., Menten, K.M., Serabyn, E., \& Zylka, R. 1994, ApJ, 423, L39 
Lis, D.C., Serabyn, E., Keene, J., Dowell, C.D., Benford, D.J., Phillips, T.G., Hunter, T.R., \& Wang, N. 1998, ApJ, 509, 299

Mangum, J.G., \& Wootten, A. 1993, ApJS, 89, 123

Martín-Pintado, J., Bachiller, R., \& Fuente, A. 1997, A\&A, 54, 315

Martín-Pintado, J., de Vicente, P., Fuente, A. \& Planesas, P. 1997, ApJ, 482, L45

Martín-Pintado, J., de Vicente, P., Rodriguez-Fernández, N.J., Fuente, A. \& Planesas, P. 2000, A\&A, 356, L8

Mathis, J.S., Mezger, P.G., \& Panagia, N. 1983, A\&A, 128, 212

Mehringer, D.M., Palmer, P., \& Goss, W.M. 1993, ApJ, 402, L69

Mezger, P.G., Duschl, W.J., \& Zylka, R. 1996, A\&AR, 7, 289

Morris, M. 1989, in The Center of the Galaxy, ed. M. Morris (Dordrecht: Kluwer), 171

Morris, M. 1993, ApJ, 408, 496

Morris, M., \& Serabyn, E. 1996, ARAA, 34, 645

Panagia, N. 1973, AJ, 78, 929

Pierce-Price, D., Richer, J.S., Greaves, J.S. et al. 2000, ApJ, submitted

Rieke, G.H., Rieke, M..J., \& Paul, A.E. 1989, ApJ, 336, 752

Serabyn, E., \& Güsten, R. 1991, A\&A, 242, 376

Serabyn, E., Shupe, D., \& Figer, D.F. 1998, Nature, 394, 448

Simpson, J.P., Colgan, S.W., Cotera, A.S., Erickson, E.F., Haas, M.R., Morris, M., \& Rubin, R.H. 1997, ApJ, 487, 689

Spergel, D. N., \& Blitz, L. 1992, Nature, 357, 665

Swinyard, B.M. et al. 1996, A\&A, 315, L43

Taylor, G.B., Morris, M., \& Schulman, E. 1993, AJ, 106, 1978

Wolfire, M.G., Tielens, A.G.G.M., \& Hollenbach, D. 1990, ApJ, 358, 116 
Table 1: Molecular observations log.

\begin{tabular}{lccc}
\hline Transition & Telescope & Date & $\eta$ \\
\hline${ }^{13} \mathrm{CO}(1-0)$ & IRAM & May 94 & 0.63 \\
${ }^{13} \mathrm{CO}(2-1)$ & IRAM & May 94 & 0.41 \\
$\mathrm{CS}(2-1)$ & IRAM & March 93 & 0.63 \\
$\mathrm{CS}(3-2)$ & IRAM & May 94 & 0.55 \\
$\mathrm{CS}(2-1)$ & CSO & April 00 & 0.75 \\
$\mathrm{C}^{34} \mathrm{~S} 2(2-1)$ & IRAM & May 94 & 0.63 \\
$\mathrm{C}^{34} \mathrm{~S} 2(3-2)$ & IRAM & March 93 & 0.55 \\
$\mathrm{C}^{34} \mathrm{~S} 2(5-4)$ & IRAM & May 94 & 0.41 \\
$\mathrm{H}_{2} \mathrm{CO}\left(3_{03}-2_{02}\right)$ & CSO & April 00 & 0.75 \\
$\mathrm{H}_{2} \mathrm{CO}\left(3_{22}-2_{21}\right)$ & CSO & April 00 & 0.75 \\
$\mathrm{H}_{2}^{13} \mathrm{CO}\left(3_{03}-2_{02}\right)$ & CSO & June 00 & 0.75 \\
$\mathrm{CH} \mathrm{OH}_{3}\left(4_{23}-3_{13}\right)$ & CSO & April 00 & 0.75 \\
$\mathrm{SiO}(5-4)$ & CSO & April 00 & 0.75 \\
$\mathrm{SO}\left(5_{6}-4_{5}\right)$ & CSO & April 00 & 0.75 \\
\hline
\end{tabular}

Table 2: Continuum source parameters.

\begin{tabular}{ccccccccc}
\hline Position & $T_{1}$ & $T_{2}$ & $\tau_{2} /\left(\tau_{1}+\tau_{2}\right)$ & $T_{d}$ & $\beta$ & $S_{350}$ & $N_{\mathrm{H}_{2}}$ & $n_{\mathrm{H}_{2}}$ \\
\hline a & 17 & 35 & $3.8 \times 10^{-2}$ & 18 & 2.8 & 520 & $2.9 \times 10^{24}$ & $3 \times 10^{5}$ \\
b & 22 & 43 & $6.3 \times 10^{-3}$ & 22 & 2.2 & 160 & $0.6 \times 10^{24}$ & $6 \times 10^{4}$ \\
c & 20 & 35 & $2.3 \times 10^{-2}$ & 20 & 2.4 & 280 & $1.0 \times 10^{24}$ & $3 \times 10^{5}$ \\
d & 17 & 32 & $3.2 \times 10^{-2}$ & 17 & 2.6 & 300 & $1.7 \times 10^{24}$ & $2 \times 10^{5}$ \\
e & 17 & 34 & $3.1 \times 10^{-2}$ & 17 & 2.8 & 610 & $3.4 \times 10^{24}$ & $4 \times 10^{5}$ \\
f & 13 & 27 & $1.3 \times 10^{-1}$ & 15 & 3.4 & 430 & $2.4 \times 10^{24}$ & $3 \times 10^{5}$ \\
\hline
\end{tabular}

Notes: Entries in the table are: position (see Fig. 1), temperatures of the two components in the gray-body fit $(\mathrm{K})$, the ratio of the optical depth of the warmer component to the total optical depth, the mean dust temperature weighted by the optical depth $(\mathrm{K})$, and the grain emissivity exponent obtained from two-component gray-body fits to the observed SED, peak $350 \mu \mathrm{m}$ flux density in a $30^{\prime \prime}$ beam (based on the data of Dowell et al. 1999), peak $\mathrm{H}_{2}$ column density assuming a dust temperature of $20 \mathrm{~K}$ and the corresponding volume density, assuming a line-of-sight depth of $1.25 \mathrm{pc}$ for source ' $\mathrm{c}$ ' and $3.0 \mathrm{pc}$ for the remaining sources. These line-of-sight depths are approximate FWHM minor axis of the observed $350 \mu \mathrm{m}$ flux distribution. Since we assume that the clouds have prolate shapes, the resulting volume densities should be considered upper limits. 
Table 3: Observed $\mathrm{H}_{2} \mathrm{CO}$ line parameters.

\begin{tabular}{cccrcc}
\hline Position & $\alpha_{1950}$ & $\delta_{1950}$ & $\mathrm{I}\left(3_{03}-2_{02}\right)$ & $\mathrm{I}\left(3_{22}-2_{21}\right)$ & $\mathrm{R}$ \\
\hline $\mathrm{a}$ & $17^{\mathrm{h}} 42^{\mathrm{m}} 59^{\mathrm{a}} 8$ & $-28^{\circ} 41^{\prime} 41^{\prime \prime}$ & $10.22 \pm 0.16$ & $3.26 \pm 0.17$ & $3.13 \pm 0.17$ \\
$\mathrm{C}$ & $17^{\mathrm{h}} 43^{\mathrm{m}} 11^{\mathrm{s}} .8$ & $-28^{\circ} 34^{\prime} 27^{\prime \prime}$ & $5.23 \pm 0.24$ & $1.69 \pm 0.27$ & $3.09 \pm 0.51$ \\
$\mathrm{e}$ & $17^{\mathrm{h}} 43^{\mathrm{m}} 37^{\mathrm{s}} 3$ & $-28^{\circ} 30^{\prime} 55^{\prime \prime}$ & $7.93 \pm 0.19$ & $1.94 \pm 0.18$ & $4.09 \pm 0.39$ \\
$\mathrm{f}$ & $17^{\mathrm{h}} 43^{\mathrm{m}} 33^{\mathrm{s}} \cdot 7$ & $-28^{\circ} 29^{\prime} 10^{\prime \prime}$ & $6.84 \pm 0.18$ & $2.10 \pm 0.18$ & $3.26 \pm 0.29$ \\
\hline
\end{tabular}

NOTES: Entries in the table are: position (see Fig. 1), observed integrated intensities of the $\mathrm{H}_{2} \mathrm{CO}$ $\left(3_{03}-2_{02}\right)$ and $\left(3_{22}-2_{21}\right)$ lines $\left(\mathrm{K} \mathrm{km} \mathrm{s}^{-1}\right.$; corrected for the main beam efficiency of $\left.75 \%\right)$ with the corresponding $1 \sigma$ uncertainties, and the resulting line intensity ratio with its uncertainty. The line uncertainties are statistical uncertainties based on the noise in the spectrum and do not include the calibration uncertainty, which is $\sim 15 \%$. Since the two lines were observed in a single spectrum, the calibration uncertainty does not affect the observed line ratio. Position ' $a$ ' corresponds to GCM0.25+0.01.

Table 4: Observed CS and $\mathrm{C}^{34} \mathrm{~S}$ line parameters.

\begin{tabular}{lrc}
\hline Transition & Intensity & FWHM \\
\hline CS (2-1) & 124 & 40 \\
$\mathrm{CS}(3-2)$ & 85 & 37 \\
$\mathrm{CS}(5-4)$ & 21 & 30 \\
$\mathrm{C}^{34} \mathrm{~S}(2-1)$ & 10.5 & 35 \\
$\mathrm{C}^{34} \mathrm{~S}(3-2)$ & 7.4 & 29 \\
$\mathrm{C}^{34} \mathrm{~S}(5-4)$ & 1.2 & 29 \\
\hline
\end{tabular}

NOTES: Entries in the table are: transition, integrated line intensity $\left(\mathrm{K} \mathrm{km} \mathrm{s}^{-1}\right)$ corrected for the corresponding main beam efficiency, FWHM linewidth $\left(\mathrm{km} \mathrm{s}^{-1}\right)$. The uncertainties are $\sim 40 \%$ for $\mathrm{C}^{34} \mathrm{~S}(5-4)$ and $15 \%$ for the remaining transitions.

Table 5: Magnitudes and colors of the IR sources in GCM0.25+0.01.

\begin{tabular}{crrrrrr}
\hline Star & $\mathrm{J}$ & $\mathrm{H}$ & $\mathrm{K}$ & $4 \mu \mathrm{m}$ & $\mathrm{J}-\mathrm{H}$ & $\mathrm{H}-\mathrm{K}$ \\
\hline 1 & 13.60 & 10.42 & 8.93 & 7.47 & 3.18 & 1.49 \\
2 & 14.37 & 11.51 & 9.96 & 9.36 & 2.86 & 1.55 \\
3 & 15.69 & 12.83 & 11.20 & 10.74 & 2.86 & 1.53 \\
\hline
\end{tabular}

Note: Positions of the three stars are marked in Fig. 13, H-band panel. 
Fig. 1.- Gray-scale image of the $173 \mu \mathrm{m}$ continuum emission in the region between the radio Arc and Sgr B1 observed with ISO LWS at $\sim 75^{\prime \prime}$ angular resolution. White contours show the distribution of the $350 \mu \mathrm{m}$ continuum emission (Dowell et al. 1999) convolved to $30^{\prime \prime}$ angular resolution. Letters ' $a$ '-' $f$ ' mark the location of the positions at which the spectral energy distributions are analyzed (Fig. 4 and Table 2). Note that the southern part of the Dust Ridge has the same velocity as the molecular cloud interacting with the non-thermal filaments in the radio Arc, indicating that these sources are likely located in the Galactic center region. Stars mark the locations of known $\mathrm{H}_{2} \mathrm{O}$ masers in the region.

Fig. 2.- Gray-scale image of the $45 \mu \mathrm{m}$ continuum emission in the region between the radio Arc and Sgr B1 observed with ISO LWS at $75^{\prime \prime}$ angular resolution. Contours and symbols are the same as in Fig. 1. Note that the GMCs are no longer seen in emission at $45 \mu \mathrm{m}$.

Fig. 3.- Distribution of the $45 / 173 \mu \mathrm{m}$ color temperature in the Ridge calculated assuming a grain emissivity exponent $\beta=1$ (appropriate for the diffuse dust component, but not for the dense cores; gray-scale and thin white contours). Black contours and symbols are the same as in Fig. 1. All submillimeter sources appear as local minima in the color temperature. The coldest dust is located in the northern part of the Sgr B1 core (position ' $f$ ' in the map). Color temperature contour levels are from 23 to $38 \mathrm{~K}$ with an interval of $3 \mathrm{~K}$.

Fig. 4.- Observed continuum spectral energy distributions between 45 and $800 \mu \mathrm{m}$ at positions 'a'-'f' (Figs. 1-3).

Fig. 5. - (Upper) The effects of the external radiation field intensity and the internal luminosity on the model SED. The SED of a cloud without strong external heating (solid line; $G_{0}=40$, $L_{o}=2.5 \times 10^{5} \mathrm{~L}_{\odot}$ ) is too narrow and grossly underestimates the observed flux at MIR wavelengths $(\lambda<100 \mu \mathrm{m})$. Models with strong external heating, but no internal heating sources (dashed line; $G_{o}=500, L_{o}=0$ ) produce a broad SED, but underestimate the flux at the wavelengths near $100 \mu \mathrm{m}$. (Middle) The best fit model including both external and internal heating (solid line; $\left.G_{o}=500, L_{o}=1.9 \times 10^{5} \mathrm{~L}_{\odot}\right)$. The dotted lines, corresponding to $L_{o}=1.0 \times 10^{5} \mathrm{~L}_{\odot}$ and $2.7 \times 10^{5} \mathrm{~L}_{\odot}$, represent the allowed range of $L_{o}$ consistent with the calibration uncertainties; the maximum central luminosity consistent with the data is equivalent to $\sim 3$ B0 ZAMS stars. (Bottom) The effect of $G_{o}$ on the model SED. The solid line is the best fit model $\left(G_{o}=500\right.$, $\left.L_{o}=1.5 \times 10^{5} \mathrm{~L}_{\odot}\right)$. The dotted lines, corresponding to $G_{o}=400$ and 600 , represent the range of $G_{o}$ consistent with the calibration uncertainties.

Fig. 6.- The CII/OI intensity ratio as a function of (CII+OI+SiII)/FIR for a grid of PDR models with a range of hydrogen densities and UV field intensities (from Wolfire et al. 1990). Gray squares correspond to several lines of sight in our ISO maps away from the submillimeter continuum sources.

Fig. 7.- Observed spectra of $\mathrm{H}_{2} \mathrm{CO}\left(3_{03}-2_{02}\right)$ and $\left(3_{22}-2_{21}\right)$ (dotted and solid lines, respectively). The two lines are observed in the same spectrum. The emission has been corrected for the main 
beam efficiency of $75 \%$. The $\mathrm{H}_{2} \mathrm{CO}$ emission occurs at LSR velocities $20-50 \mathrm{~km} \mathrm{~s}^{-1}$ and the strong line at LSR velocities $50-100 \mathrm{~km} \mathrm{~s}^{-1}$ is the $\left(4_{31}-3_{21}\right)$ transition of $\mathrm{CH}_{3} \mathrm{OH}$ at $218.440047 \mathrm{GHz}$.

Fig. 8.- Molecular spectra toward GCM0.25+0.01: (upper) ${ }^{13} \mathrm{CO}(1-0)$ and (2-1) (solid and dotted lines, respectively), (middle) CS (2-1), (3-2), and (5-4) (solid, dotted, and dashed lines respectively), (lower) $\mathrm{C}^{34} \mathrm{~S}(2-1),(3-2)$, and (5-4) (solid, dotted, and dashed lines respectively). The intensity scale is the main beam brightness temperature.

Fig. 9.- A grid of LVG models for CS and $\mathrm{C}^{34} \mathrm{~S}$ as a function of gas density and kinetic temperature. The gray-scale and black contours correspond to $\chi^{2}$ for the $\mathrm{C}^{34} \mathrm{~S}$ models. Contour levels are 4.5, 5.5, 10 , and 60 . White contour correspond to the CS models. Contour levels are 1,3 , and 30 . The cross shows the range of densities in GCM0.25+0.01 implied by our CS/ $\mathrm{C}^{34} \mathrm{~S}$ data and temperatures consistent with the observed $\mathrm{H}_{2} \mathrm{CO}$ line ratio.

Fig. 10. - Channel maps of ${ }^{13} \mathrm{CO}(2-1)$ emission in GCM0.25+0.01. Channel width is $10 \mathrm{~km} \mathrm{~s}^{-1}$ and the center velocities are given in the upper right corner of each panel. Contour levels are from 2 to $6 \mathrm{~K} \mathrm{~km} \mathrm{~s}^{-1}$, spaced by $1 \mathrm{~K} \mathrm{~km} \mathrm{~s}^{-1}$. Symbols mark the location of the $\mathrm{H}_{2} \mathrm{O}$ maser (star) and the SiO peak (square). White contour in the $10 \mathrm{~km} \mathrm{~s}^{-1}$ panel outlines the location of the $350 \mu \mathrm{m}$ continuum emission.

Fig. 11. - Channel maps of CS (3-2) emission in GCM0.25+0.01. Channel width is $10 \mathrm{~km} \mathrm{~s}^{-1}$ and the center velocities are given inn the upper right corner of each panel. Contour levels are from 1 to $3.5 \mathrm{~K} \mathrm{~km} \mathrm{~s}^{-1}$, spaced by $0.5 \mathrm{~K} \mathrm{~km} \mathrm{~s}^{-1}$. Symbols and the white contour are the same as in Fig. 10 .

Fig. 12. - Spectra of $\mathrm{SiO}(5-4)$ and SO $\left(5_{6}-4_{5}\right)$ toward GCM $0.25+0.01$. Offsets are in seconds of arc with respect to the reference position $\alpha_{1950}=17^{\mathrm{h}} 42^{\mathrm{m}} 58 \mathrm{sec}, \delta_{1950}=-28^{\circ} 41^{\prime} 05^{\prime \prime}$. The offsets $(24,-36)$ correspond to the position ' $a$ ' in Table 3 . The spectrum in the upper-right corner corresponds to the position ' $\mathrm{e}$ ' in Table 3.

Fig. 13. - NIR images of the region in GCM0.25+0.01 centered at the position of the $\mathrm{H}_{2}$ Omaser. Wavelengths are given in the upper right corner of each panel. Locations of the three stars from Table 5 are marked in the H-band panel. Contour levels are 5, 10, 15, 30, 70, 150, and 400 times the corresponding rms noise level. The square marks the expected position of the $\mathrm{H}_{2} \mathrm{O}$ maser. 
Fig. 1

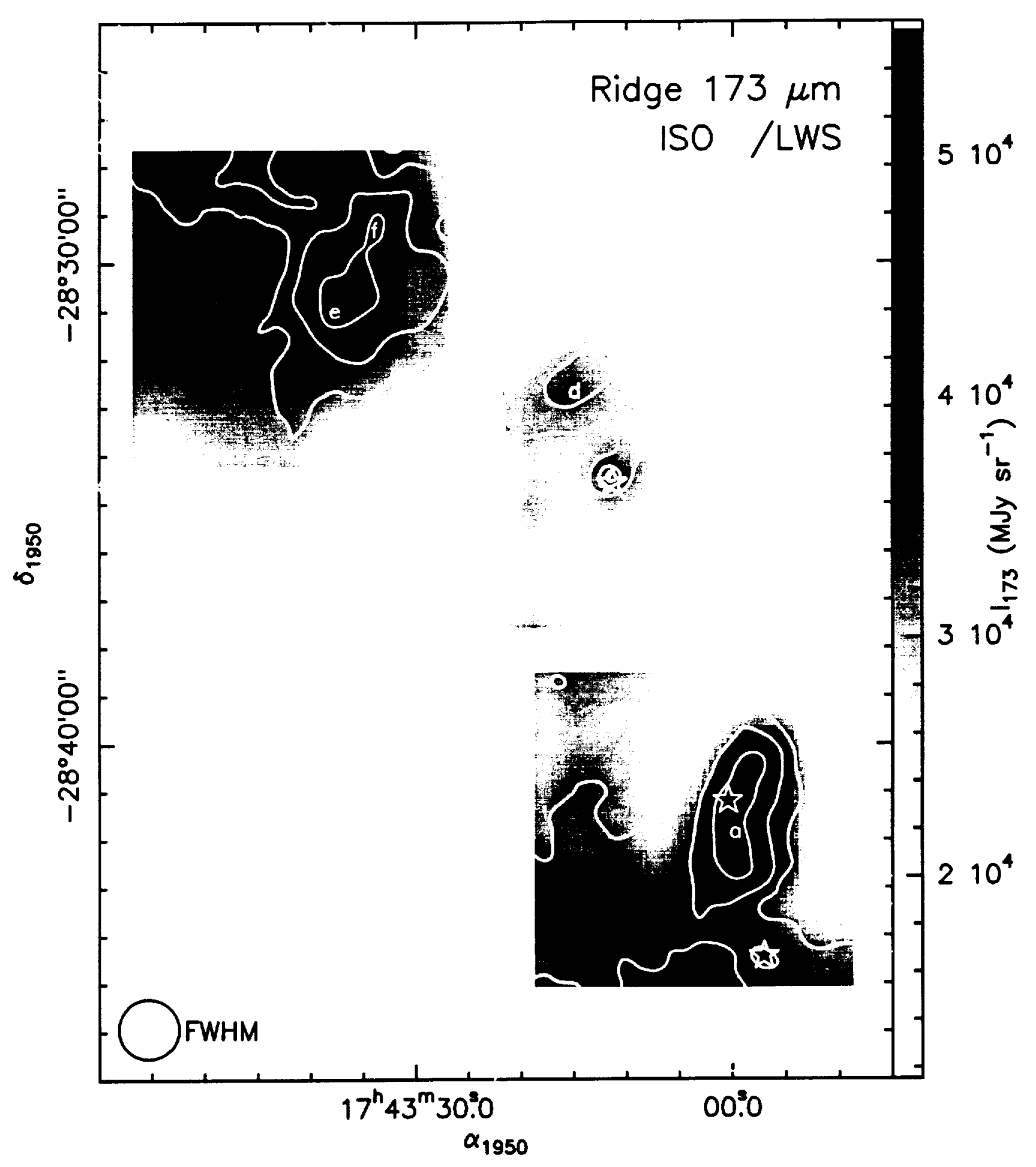


Fig. 2

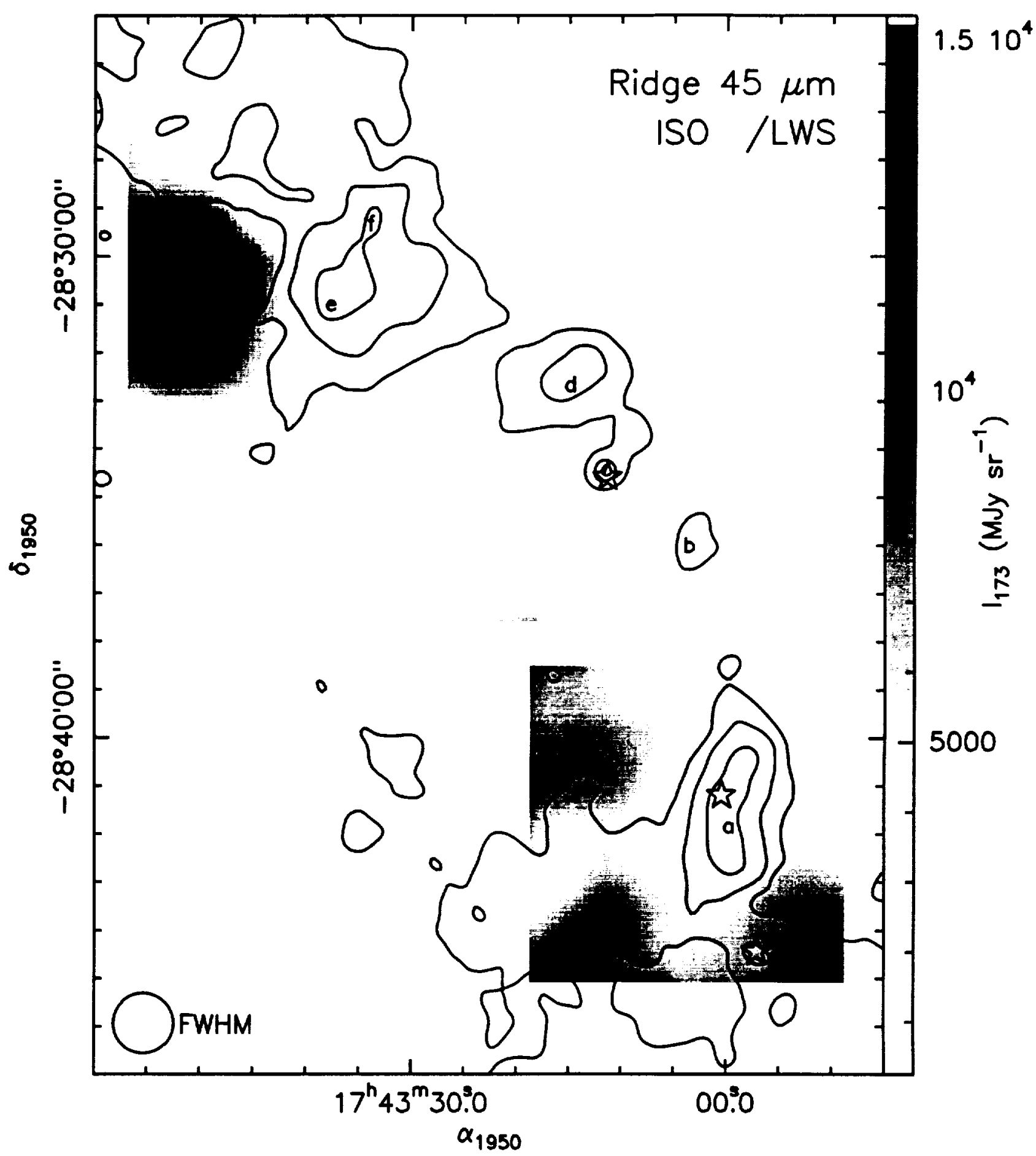


Fig. 3

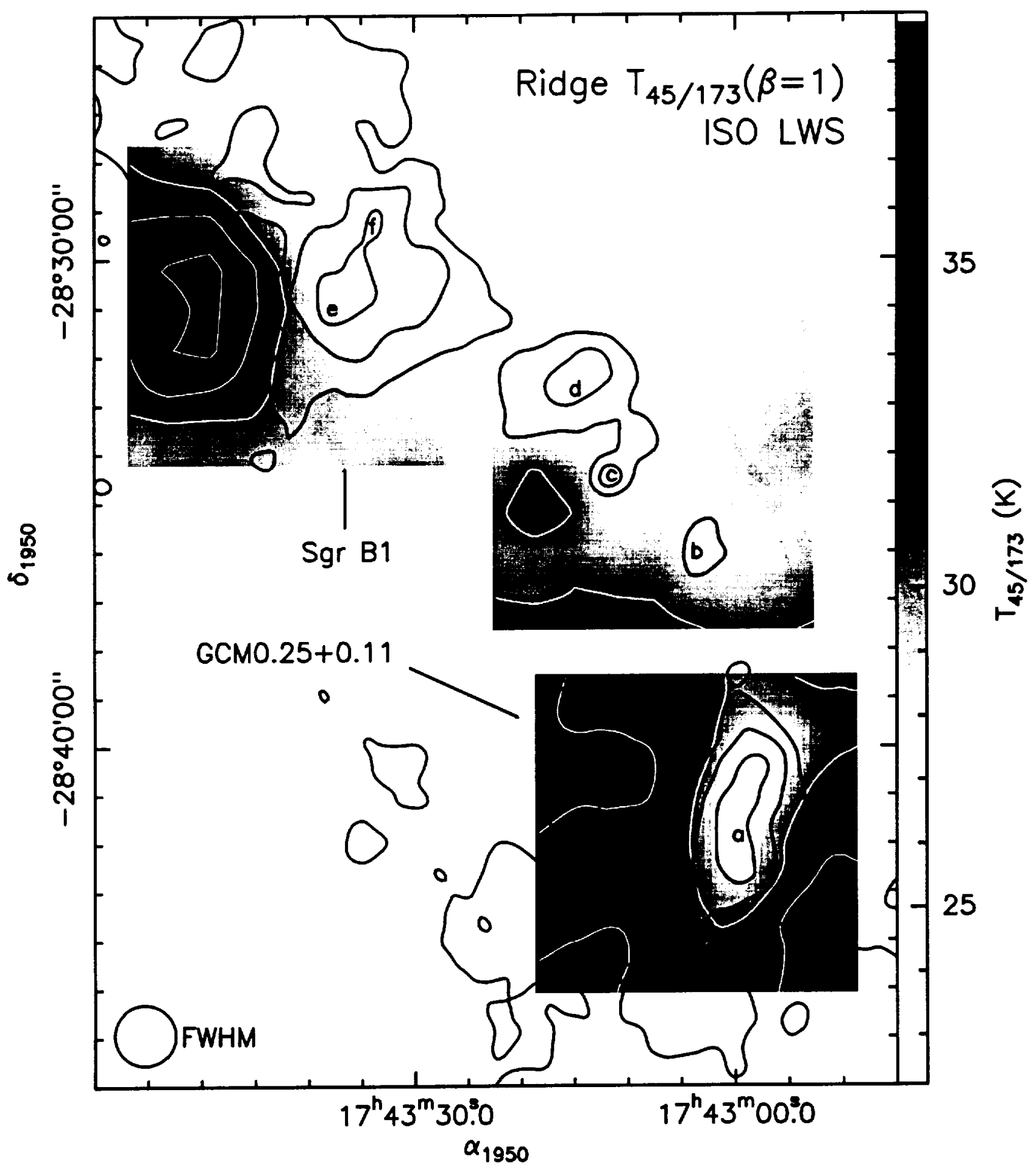


Fig. 4

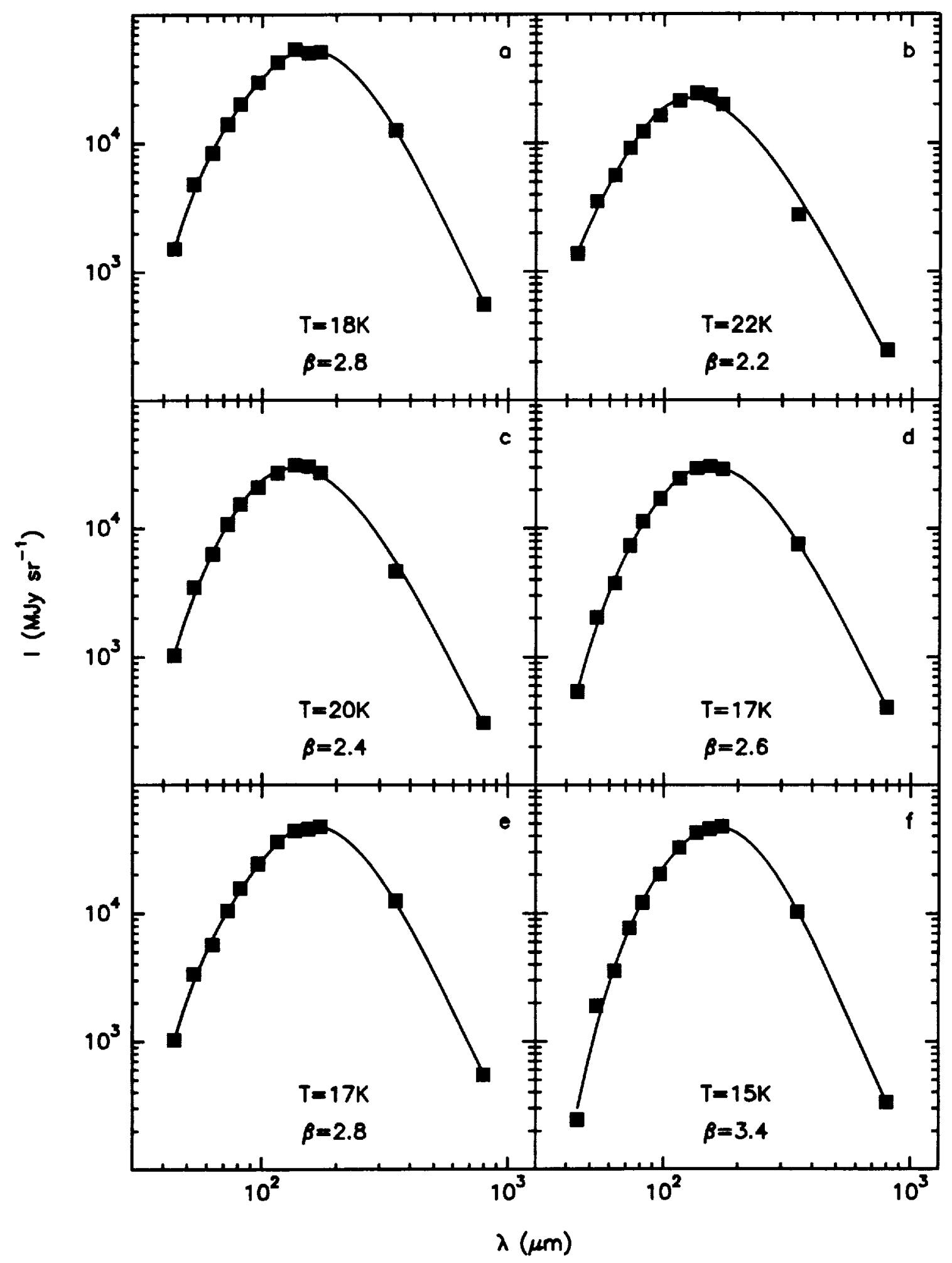


Fig. 5

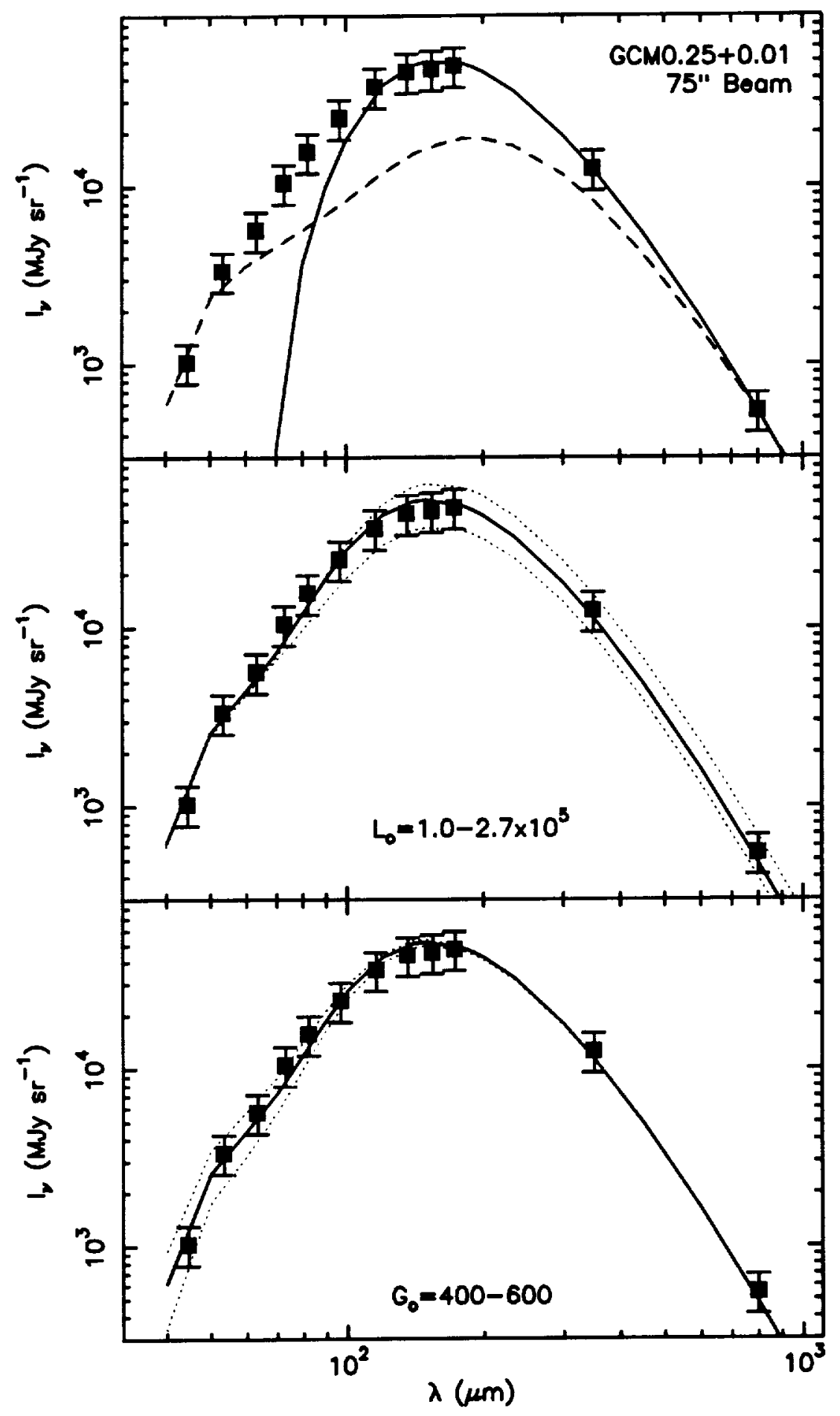


Fig. 6

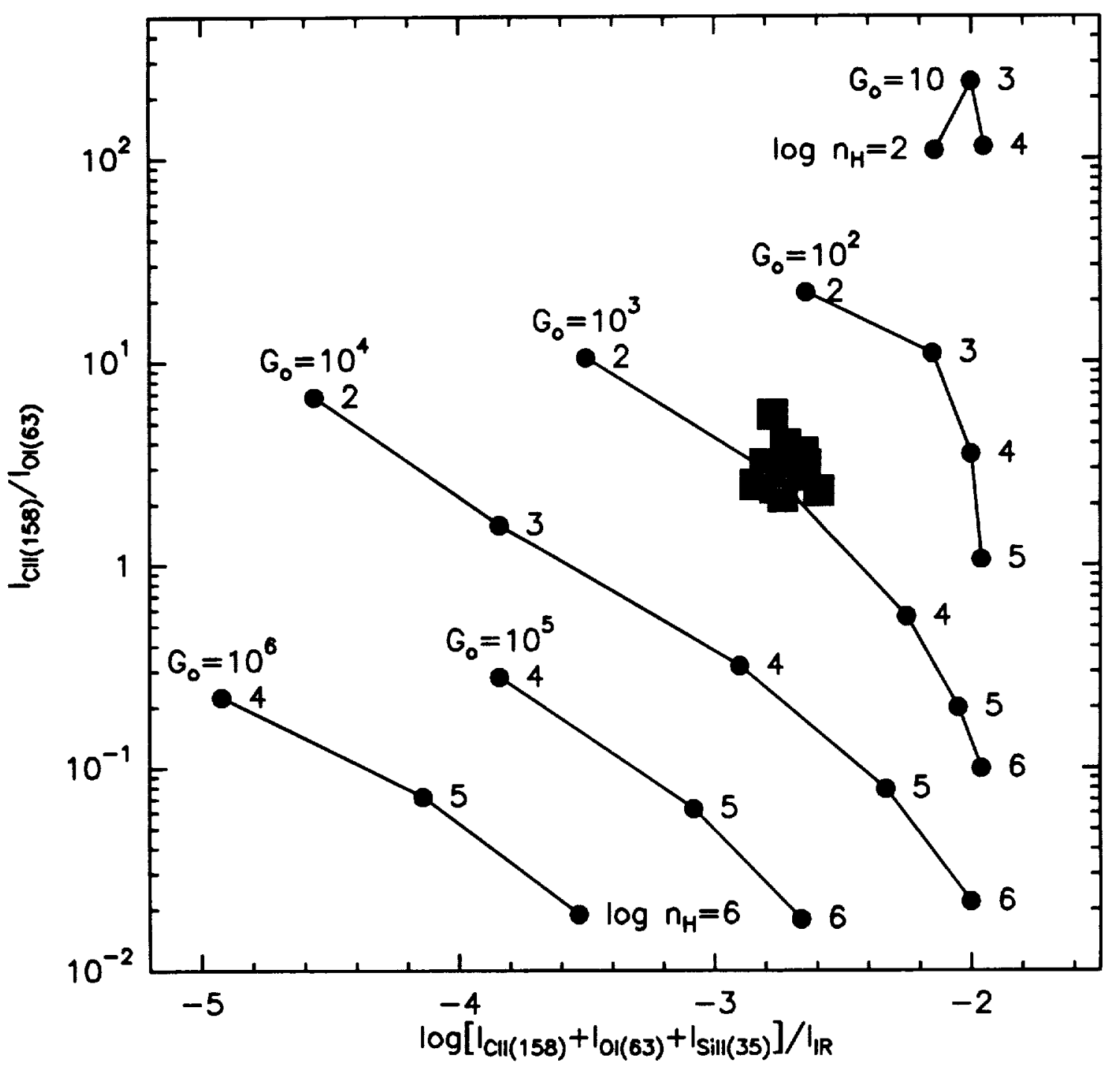


Fig. 7

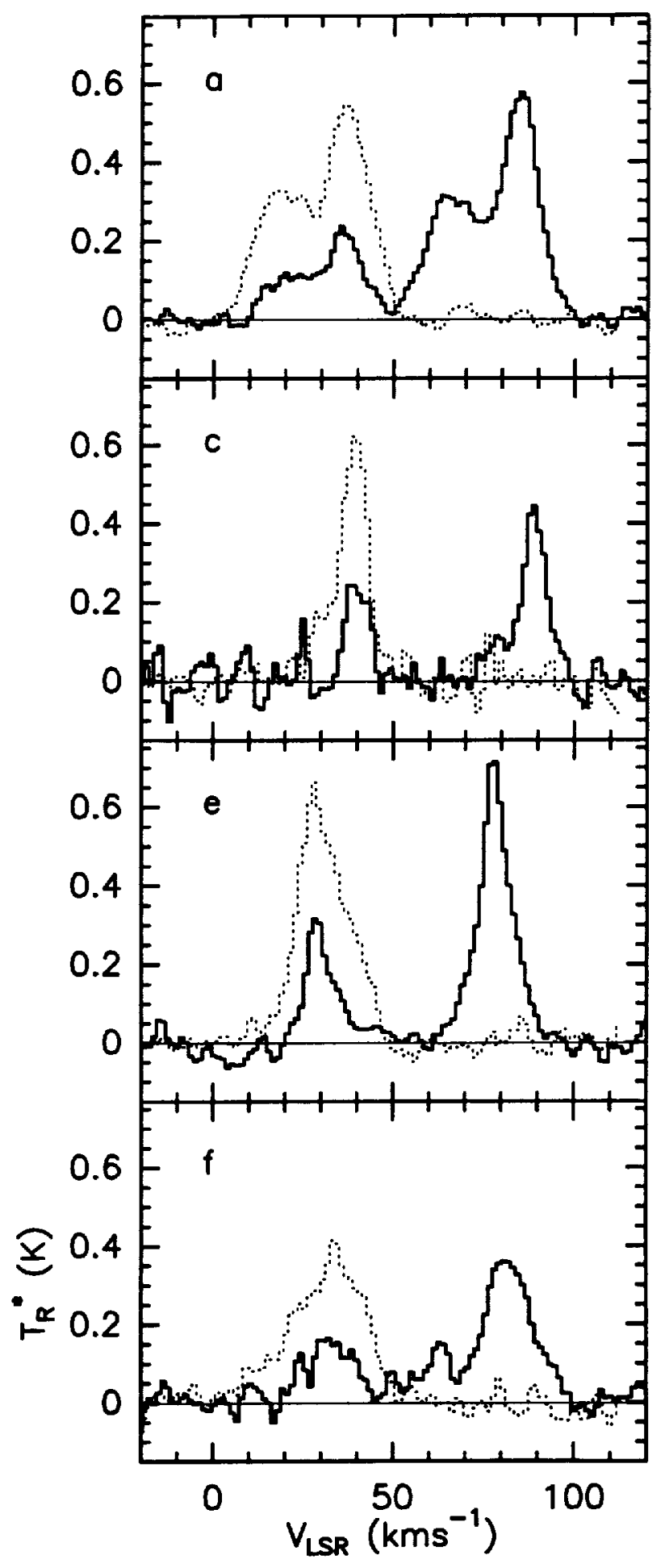


$-28-$

Fig. 8

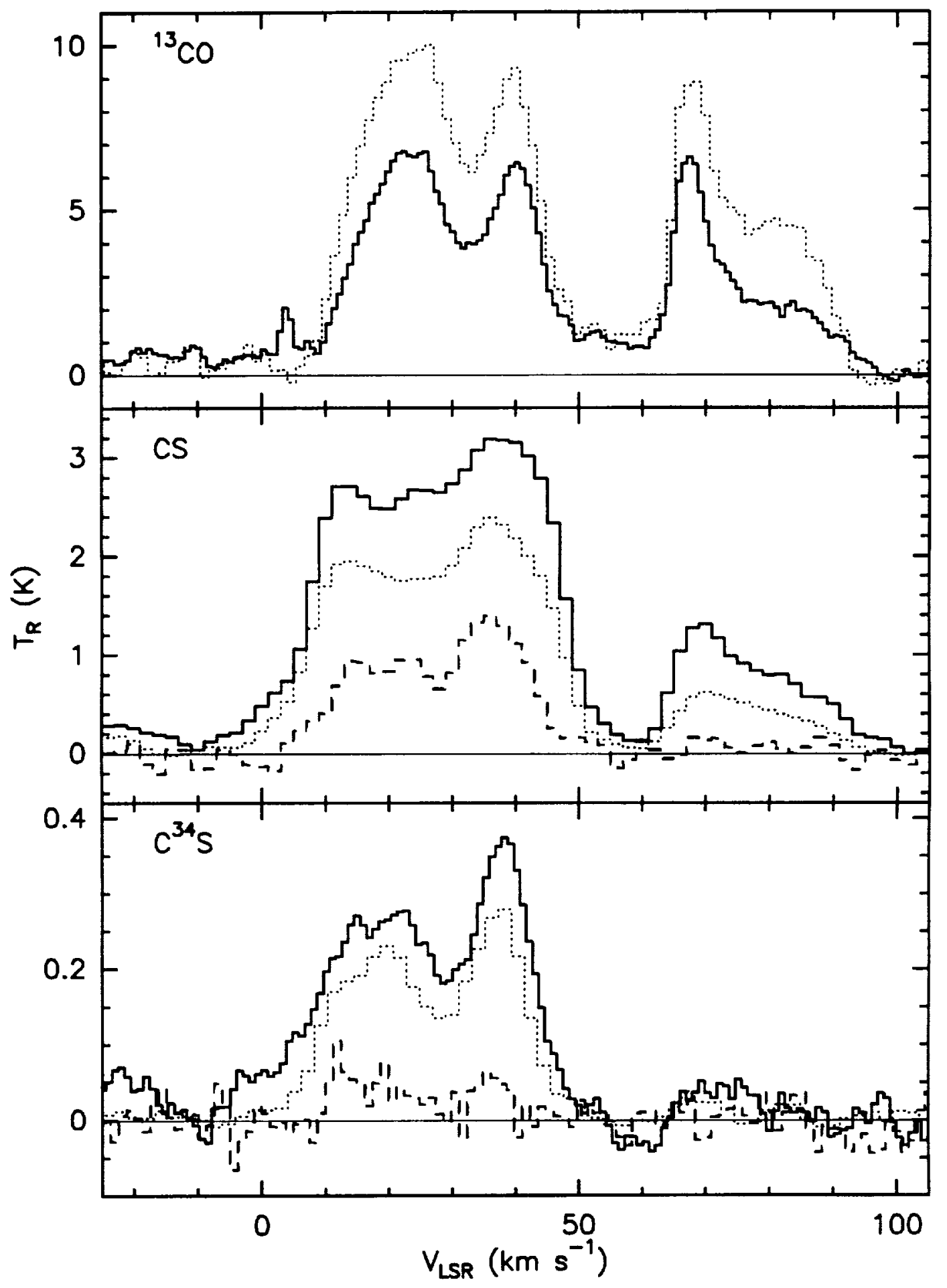


Fig. 9

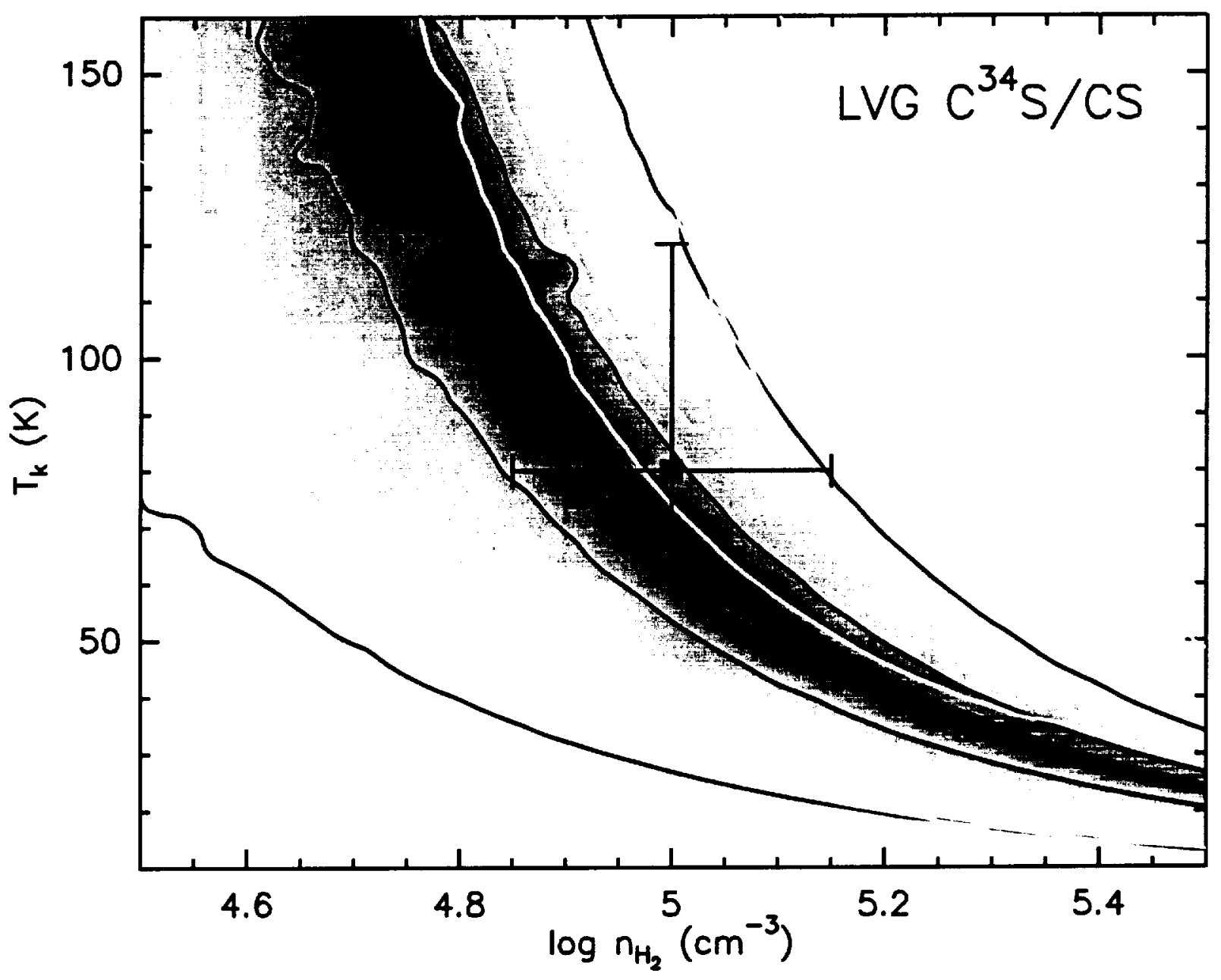


Fig. 10

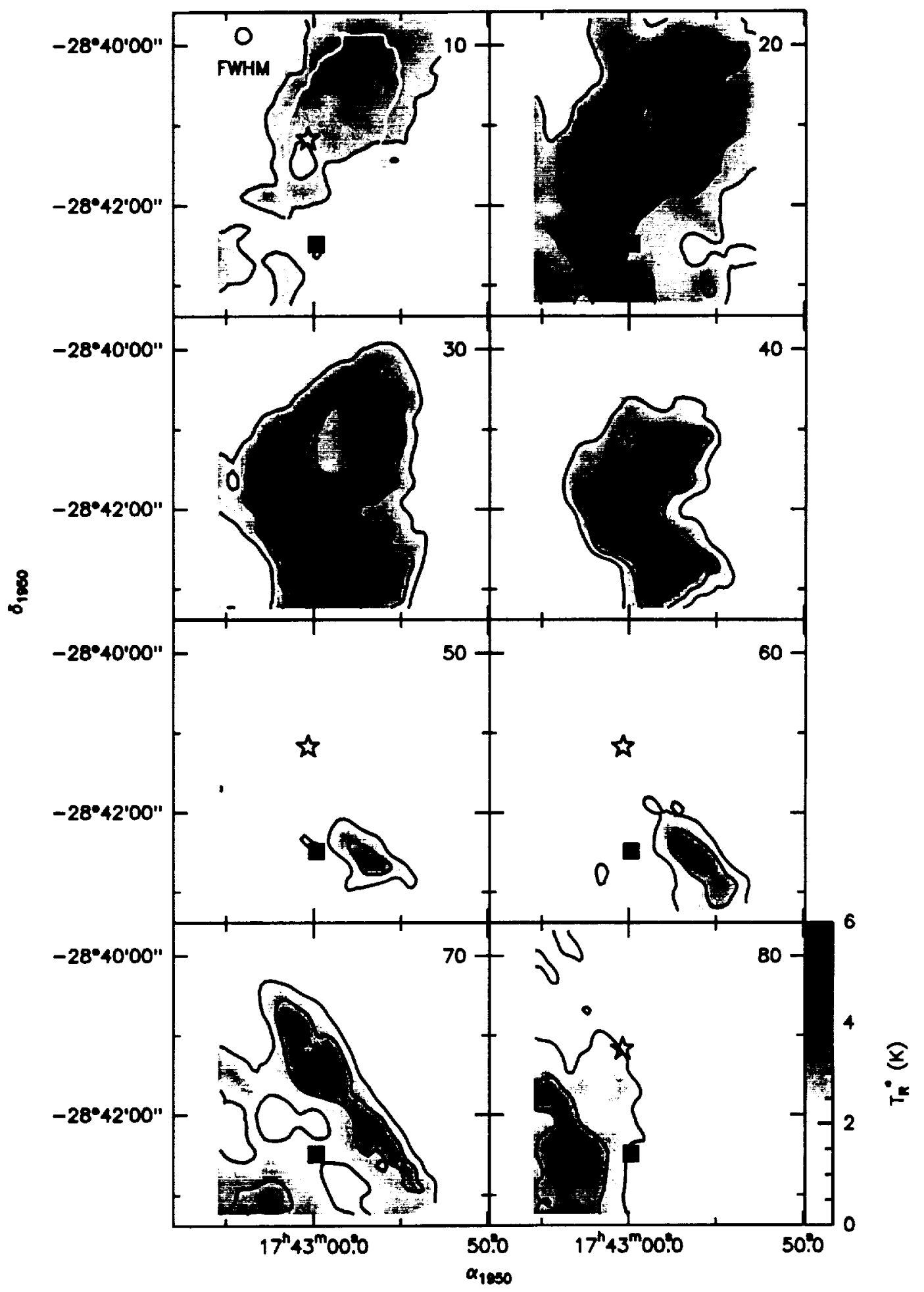


Fig. 11

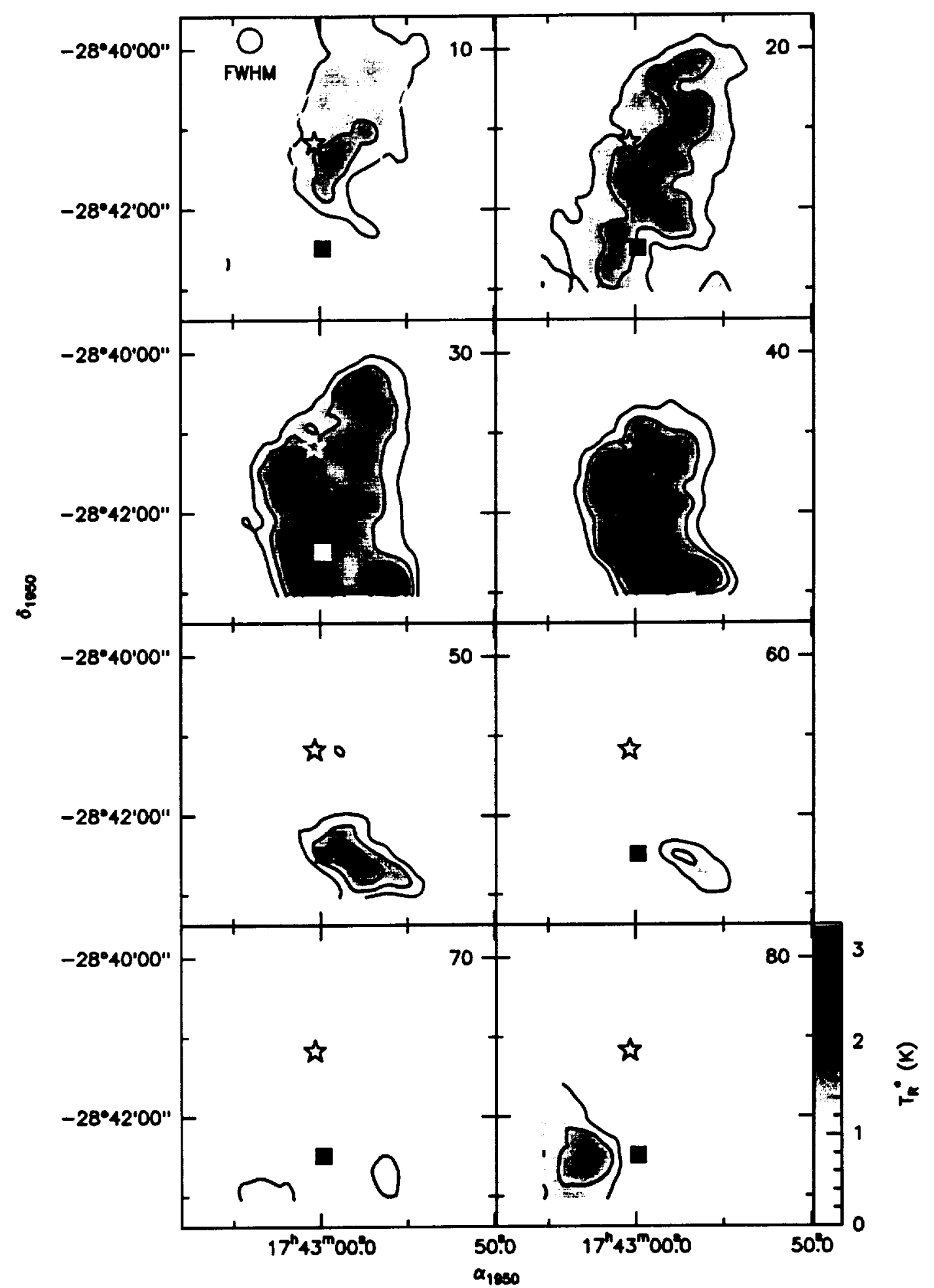


Fig. 12

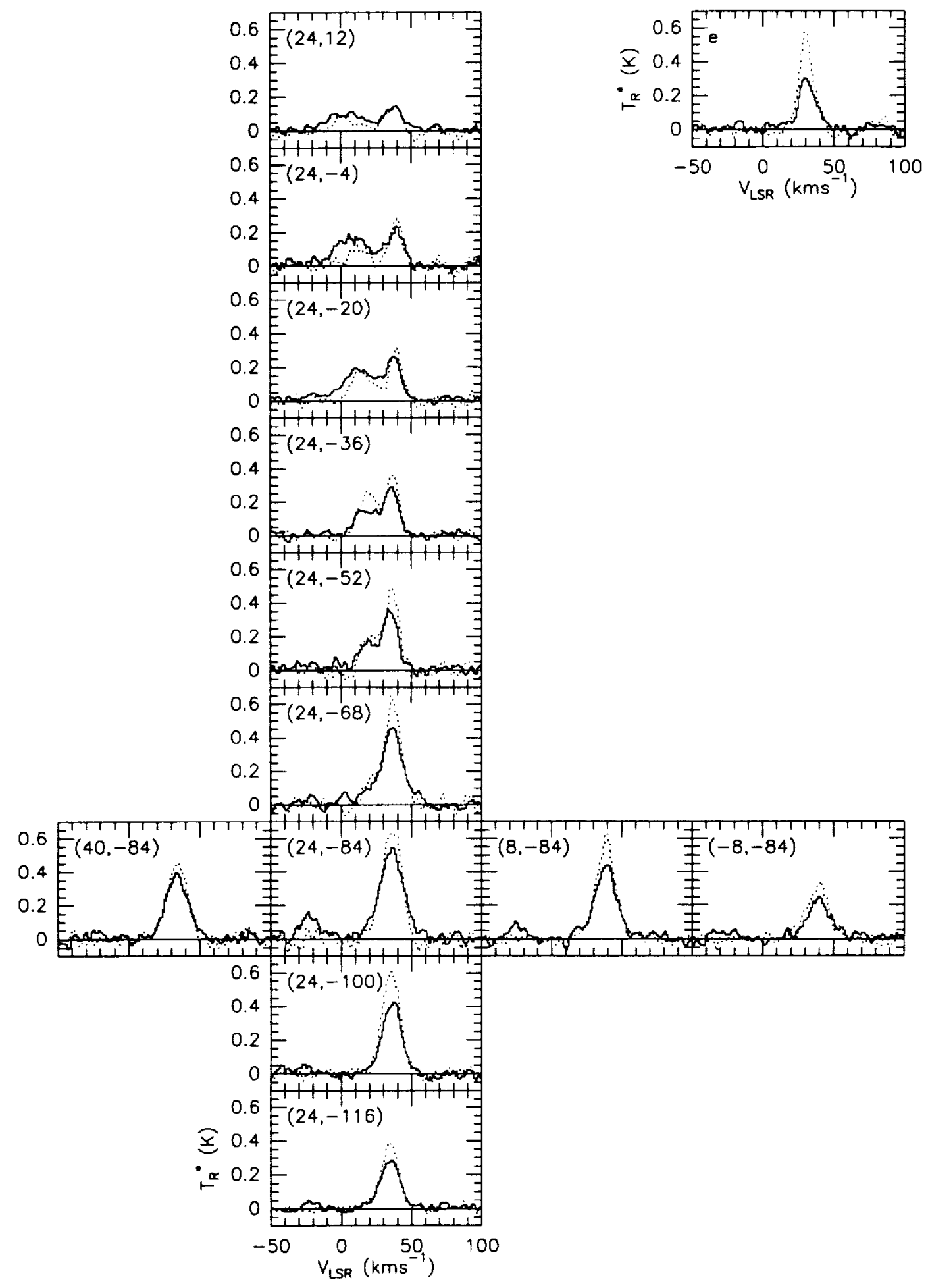


Fig. 13

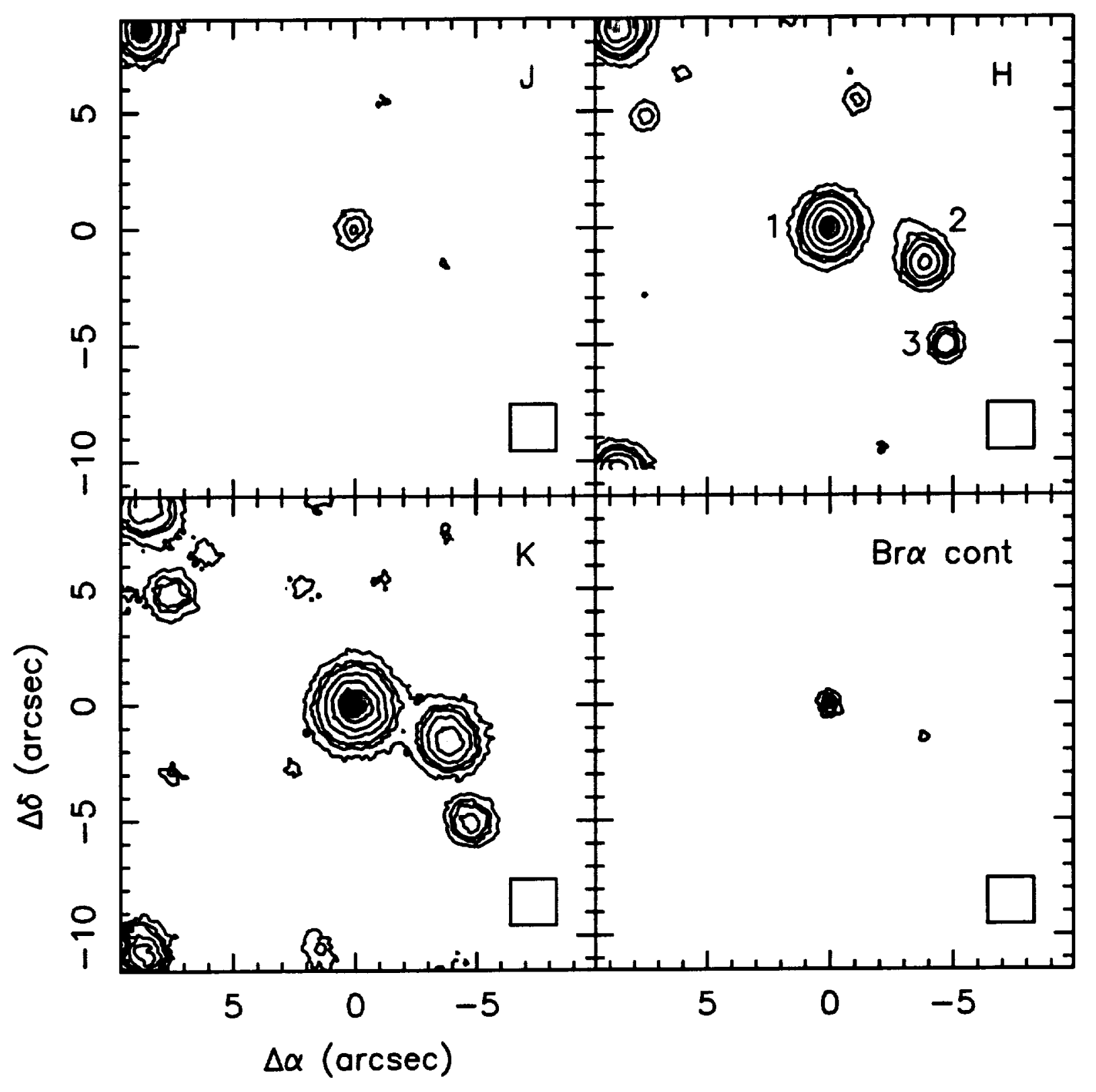


